\title{
Comparison between three optimization methods for the minimization of maximum bending load and springback in wiping die bending obtained by an experimental approach
}

\author{
Riadh Bahloul • Lanouar Ben Ayed • Alain Potiron • \\ Jean-Luis Batoz
}

Received: 28 September 2008 / Accepted: 21 September 2009 /Published online: 14 October 2009

(C) The Author(s) 2009. This article is published with open access at Springerlink.com

\begin{abstract}
The sheet metal bending process is widely used in the automotive industries, and it is actually one of the most important manufacturing processes. The robustness and the reliability of the bending operation, like many other forming operations, depend of several parameters (geometry, material, and process). In this paper, the die radius and the clearance between the punch and the sheet are optimised in order to reduce the maximum bending load and the springback. Two optimization problems are formulated, and three optimization procedures based on the response surface method are proposed and used to find the optimum solutions. Global and local approximations are used to replace the initial optimization problem, which is implicit by an explicit problem, and the optimum is localised using two algorithms: a sequential quadratic programming and an evolution strategies. The objective functions are evaluated experimentally into a limited points number, which are defined using a design of experiments technique. Good results are obtained from the three optimization procedures. The ability of each technique to
\end{abstract}

R. Bahloul $(\bowtie)$

LGM, Ecole Nationale d'Ingénieurs de Monastir,

Avenue Ibn Eljazzar,

5019 Monastir, Tunisia

e-mail: bahloul_riadh@yahoo.fr

A. Potiron

LPMI-ERTGI, ENSAM,

2 Boulevard du Ronceray, 49035, Angers, France

L. B. Ayed · J.-L. Batoz

(GIP-InSIC) Institut Supérieur d'Ingénierie de la Conception,

27 rue d'Hellieule,

88100 Saint-Dié-des-Vosges, France find the optimal solution is evaluated, and the results show a good agreement between those three methods.

Keywords Sheet metal bending · Optimization · Bending load $\cdot$ Springback $\cdot$ DoE $\cdot$ Response surface method $\cdot$ SQP . Evolution strategies

$\begin{array}{ll}\text { Abbreviations } & \\ \text { RSM } & \text { Response surface method } \\ \text { GAp } & \text { Global approximation } \\ \text { LA } & \text { Local approximation } \\ \text { SQP } & \text { Sequential quadratic programming } \\ \text { ES } & \text { Evolution strategies } \\ \text { DoE } & \text { Design of experiments } \\ \text { ANN } & \text { Artificial neural network } \\ \text { DACE } & \text { Design and analysis of computer } \\ & \text { experiments } \\ \text { RBF } & \text { Radial basis function } \\ \text { MLS } & \text { Moving least squares } \\ \text { PDEs } & \text { Partial differential equations } \\ \text { CMA-ES } & \text { Covariance matrix adaptation-evolution } \\ & \text { strategies } \\ \text { HSLA } & \text { High-strength low-alloyed steel } \\ \text { EP } & \text { Evolutionary programming } \\ \text { GAl } & \text { Genetic algorithms }\end{array}$

\section{Introduction}

Sheet metal bending process plays a major role in the automotive industries. The dimensional accuracy of bent components is a critical factor in these industries because it often determines a customer's impression of overall product quality. At the end of the bending operation, when the tools 
are removed, the metal tries to be turned over to its original shape because of residual forming stress, and that generates a shift between the desired geometries and that obtained. This phenomenon called "springback" depends principally on material properties of geometrical tools parameters and of process parameters. The improvement of the bent parts' conformity is crucial and leads to a reduction of assembly problems and to an improvement of performances of the final product.

In the last years, the international competition of the industries is extremely severe; all companies try to reduce manufacturing costs on the one hand and increase productivity, robustness of the forming process, and quality on the other. Experimental and numerical analyses are more and more used to evaluate the difficulties in sheet metal forming and to achieve these goals. There are numerous studies on sheet metal bending, but a small number of them deal with the process optimization. $\mathrm{Xu}$ et al. [1] analysed the behaviour of electronic packages under thermal and bending loads by means of quasi-meshless methods. This implicit problem was solved using multi-quadratic response surface, in order to approximate the response of finite element formulations for each loading condition. In each case, a response surface was created based on design of experiments (DoE) matrix using Latin hypercube sampling scheme. In 2004, Wu and Altan [2] developed an optimization procedure in order to improve the quality of a clutch hub, which was carried out using a deep-drawing process. A number of design modifications were evaluated to determine the optimum parameters for the selected process conditions. The results have been validated with observations of the actual process.

In [3], a methodology for the design of plate-forming dies in cylindrical bending using optimization techniques was developed in order to reduce the cost of die production by reducing the trial-and-error procedure in determining the final die geometry. The plate thickness is discretised by plane-strain finite elements. The die is assumed to be rigid, and its profile is approximated by Bezier curves, the control-points coordinates of which are the design varia- bles. The die profile is varied to minimise the difference between the required shape and the shape of the bent plate, considering the springback action. In their recent works, Naceur et al. [4] proposed a new numerical approach to optimise the shape of the initial blank, which plays an important role on the quality of the final 3D workpiece obtained by the deep drawing of thin sheets. This new approach was based on the coupling between the inverse approach used for the forming simulation and an evolutionary algorithm. The preliminary results dealing with the optimization of the blank contour in the case of square cup (the Benchmark test of Numisheet 1993) show the efficiency and the potential interest of the proposed approach.

Frequently, the bending process such as wiping die bending (see Fig. 1) induces manufacturing problems primarily due to the effect of process parameters on springback and on the loads applied by the tools. The influence of tools, particularly the bending die design and the punch-sheet clearance have been detailed in several works $[5,6]$, mentioning that it affects highly the bending angle value. In 2000, Inamdar et al. [7] described an artificial neural network based on the backpropagation of error. It is used to improve the sheet metal bending process in which an attempt is made in order to restrict springback and consequently to obtain the final angle of bend within a small tolerance. The architecture, established using an analytical model for training consisted of six inputs, ten hidden, and two outputs nodes (punch displacement and springback angle). The six inputs were the angle of bend, the punch radius/thickness ratio, the die gap, the die entry radius, the yield strength to Young's modulus ratio, and the strain hardening exponent, $n$. The effect of network parameters on the mean square error of prediction was studied.

As many manufacturing processes cannot be discrebed exactly by an analytical formulation, the use of design and analysis of computer experiments has drastically grown during the last decade, and several methods have been proposed for the analysis of simulation results or experiments, and a survey of the state of the art can be found in

Fig. 1 Experimental system of bending
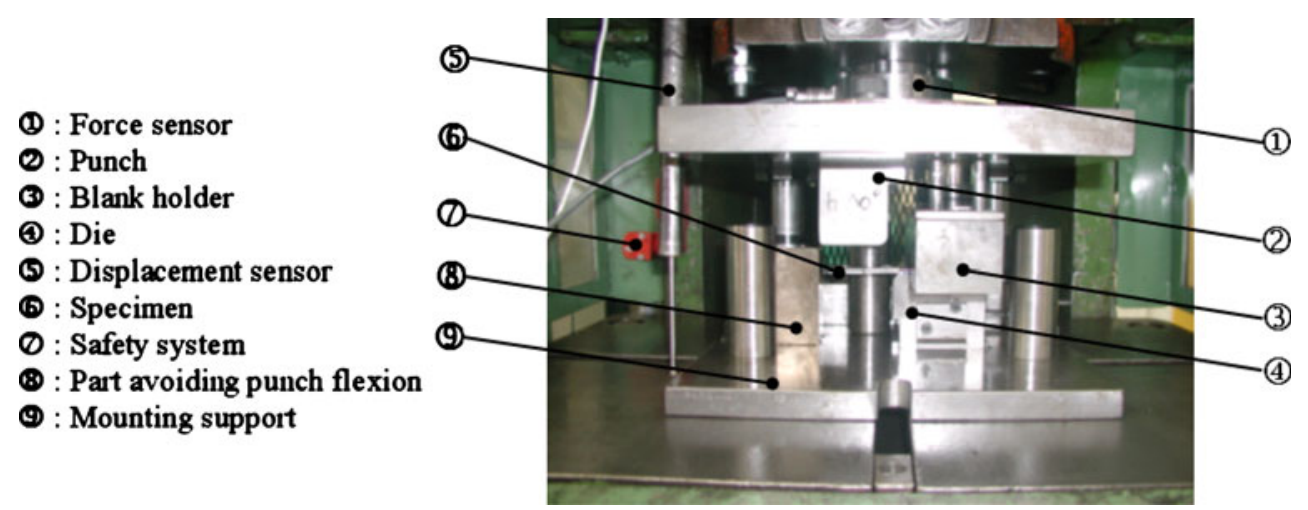
[8] by J.P.C. Kleijnen. Response surfaces based on DoE [911] Kriging methods [12] were developed for the purpose of approximating complex functions arising in different branches of applied sciences. In another work, Simpson et al. [13] compared and contrasted the use of two response surface models to approach the computer analysis: a polynomial of second order and a Kriging model. Both methods were applied to the multidisciplinary design of an aerospike nozzle which consists of a computational fluid dynamics model and a finite-elements model.

For such implicit optimizatiom problems, the approximation methods have recently been gaining attention. An optimization method using Kriging approximation is applied to an optimization problem by Sakata et al. in 2003 [14]. The methodology involves two main procedures. The first consists to finding the response surface using the Kriging method, and the second is an optimization strategy. The use of the Kriging method makes easier to perform the approximation of the cost function. Good results were obtained using the Kriging method, and they were then compared with those obtained by neural network. Several other works were carried out using the response surface method (RSM) with models and approximation strategies more or less different [15-17]. Barthelemy and Haftka [18] and Haftka and Scott [19] reported on their survey of optimization using RSM. Box and Wilson discussed and showed the reliability of the polynomial approximation in the localization of the optimal design using experimental data [20]. Hosder et al. [21] reported on the application of polynomial response surface approximation to the multidisciplinary optimization of high-speed civil transport.

RSM are well established for physical processes as documented by Myers and Montgomery [22], while the applications to simulation models in computational mechanics form a relatively young research field. Among other recent works, Roux et al. [23] discuss experimental design techniques and the regression equations for structural optimization. An approach to crashworthiness design uses genetic algorithms to select optimal set of experiments between $r^{n}$ factorial designs is described by Kurtaran et al. [24]. An application to sheet metal forming process simulated by dynamic explicit method is given by Stander [25] with an enphasis on oscillating solutions. RSM combined with stochastic finite elements are used by Kleiber et al. [26] for reliability assessment in metal forming.

The moving least squares (MLS) approximation [27] is largely used in the so-called meshfree methods for solving partial differential equations [28]. Its properties make it also useful in a range of other computational mechanics applications varying from field transfer between successive remeshing in large deformation plasticity [29] to surface interpolation for 3D adaptive mesh generators [30]. In this paper, the MLS approximation is investigated and coupled to an optimization strategy in order to find the optimum with precision and with lower cost by minimising the evaluation number of the objective function. When the DoE and the regression models are selected, any usual descent method can be used to find the optimum design.

A new approach based on augmented and compactly supported radial basis function (RBF) was developed by Krishnamurthy in 2003 [31]. The developed method was tested in two numerical examples. The response surface generated using polynomial augmented RBF predicts the response of a system better than the one constructed using classic RBF. The results obtained with the RBF-based methods were also compared with the results obtained using local methods based on MLS and Kriging. It was found that all the three local methods (RBF, MLS, and Kriging) predicted the response with almost the same accuracy. Another class of local response surface construction using $\mathrm{RBF}$ is also widely used by McDonald et al. [32].

Some papers have tried to establish a new approach for experimental optimization. They suggest using evolution strategies (ES) to sweep a region of interest and select the optimal (or near optimal) settings to a process [33]. The ES results in a highly parallel algorithm which scales favourably with large number of processors. This is accomplished by efficiently incorporating the available information from a large population, thus significantly reducing the number of generations needed to adapt the covariance matrix [34]. This optimization algorithm does not need for objective functions to be differentiable.

Our approach is based on a derandomised ES with covariance matrix adaptation (CMA-ES). The experimental results of Hansen et al. [35] have shown the advantageous convergence properties of the CMA-ES when compared to several other ES for a wide class of problems. The primary feature of the CMA-ES is its reliability in adapting an arbitrarily oriented scaling of the search space in a small populations. In particular, the algorithm is independent of any linear transformation of the coordinate system apart from the initialization.

In this paper, the determination of the optimum process parameters, such as the die radius and the clearance between the punch and the sheet, is investigated in wiping die-bending operations in order to minimise the maximum bending load and the springback obtained by an experimental approach. Two optimization problems are formulated, and DoE method is proposed for both reliability analysis and optimization process in order to properly reproduce the mains effects and interactions of the geometrical parameters of sheet forming. Mathematical models were developed for the representation of objective functions by a nonlinear regression using the least squares method. The goal of this article is to compare between three optimization procedures which are used to determine the optimum parameters: punch-sheet clearance $\mathrm{C}$ and die radius 
Table 1 Chemical composition of S500MC steel

\begin{tabular}{llllllllll}
\hline & $\mathrm{C}$ & $\mathrm{Si}$ & $\mathrm{Mn}$ & $\mathrm{P}$ & $\mathrm{S}$ & $\mathrm{Al}$ & $\mathrm{Nb}$ & $\mathrm{Ti}$ & $\mathrm{V}$ \\
\hline$(\%)$ Real & 0.09 & 0.058 & 0.91 & 0.014 & 0.002 & 0.044 & 0.056 & 0.001 & 0.043 \\
$(\%)$ Min & - & - & - & - & - & 0.01 & 0.01 & - & 0.01 \\
$(\%)$ Max & 0.12 & 0.5 & 1.7 & 0.03 & 0.03 & 0.1 & 0.08 & 0.12 & 0.08 \\
\hline
\end{tabular}

$R_{\mathrm{d}}$. The first is based on an optimization by global approach, the second on a sequential quadratic programming (SQP) algorithm, and the third on the ES. The ability of each technique to find the optimal solution is evaluated, and good results are obtained from these optimization techniques. Some conclusions are drawn from the numerical results.

\section{Experimental bending process}

The experimental tests serve as a primary information to tackle the sheet metal forming problem. They are likely to provide useful information which makes possible to establish the analytical formulations in order to optimise the process.

The development of mathematical models from a set of experimental tests was not carried out sufficiently in the case of wiping die bending process. This concerns the following two responses: the maximum bending load and springback which depends on a precise estimate of the residual stresses distribution throughout the metal sheet. For this reason, we will propose an experimental process caracterization which takes into account the damage phenomenon of material. This permits to provide a reliable experimental base.

In the remaining part of the study, we will be interested in normalised steel: type Corus. It is delivered in the form of rolled sheet of thickness $4 \mathrm{~mm}$. The material used for the fabrication of specimens is high-strength low-alloyed steel which has a designation of S500MC. The chemical composition of this material giving the percentage of carbon and the proportions of dispersoid elements are mentioned in Table 1 [36].

This study concerns the wiping die bending of specimens on mechanical press Grimar-13675 by using an instrumented punch-die system. The tools are connected to a force sensor (type: FN 300TC), to a displacement sensor (type: $D X 100$ ), and to a data acquisition system. This processing system which produces a curve for each bending operation displays the force as a function of real punch stroke. Each specimen was bent to approximately $90^{\circ}$ using bending system experiments illustrated in Fig. 1.
Lubrication of the parts and the tools before bending operation is done with a full-strength oil [37, 38]. The following relations were investigated:

Influence of clearance $(C)$ on bending load and springback;

Influence of die radius $\left(R_{\mathrm{d}}\right)$ on bending load and springback.

During the preliminary phase of the experiments, the machine tool program, the tools set-up, and measurement methods were established. In our study, a full factorial design is conducted using the following parameters:

Seven different clearances between the sheet to be bent and tool with the values varying from -0.6 to $0.6 \mathrm{~mm}$ with uniform increment of $0.2 \mathrm{~mm}$;

Four die radii having a varied values of 1,2, 4, and $6 \mathrm{~mm}$.

The initial and final configurations of specimens after bending are represented in Fig. 2. The proposed experimental investigation in the form of DoE permits to take into account all possible combinations and the effects of interactions between punch-sheet clearances and die radii values in order to optimise the process. The punch radius and the stroke are maintained constant equal to 4 and $29 \mathrm{~mm}$, respectively. They correspond to the optimal values allowing to reduce the springback [5].

The experimental procedure needed 28 cases of tests in press tools: four die radii $\times$ seven clearance values, respectively. Knowing that each test was repeated five times and therefore more than 140 bending tests were carried out. In what follows, the results obtained from the experiments on specimens of $4 \mathrm{~mm}$ thickness with punch and blank holder radius equal to $4 \mathrm{~mm}$ are retained. In this part of the study, the first cost function which was selected to constitute the response of specimens during the bending operation represents the maximum force applied by punch. The mean values of this response $F_{\text {mean }}=\frac{1}{5} \sum_{i=1}^{5} F_{i \text { measured }}$ according to the geometrical process parameters are given in Table 2.
Fig. 2 Geometries of speciemens before (a) and after (b) bending process

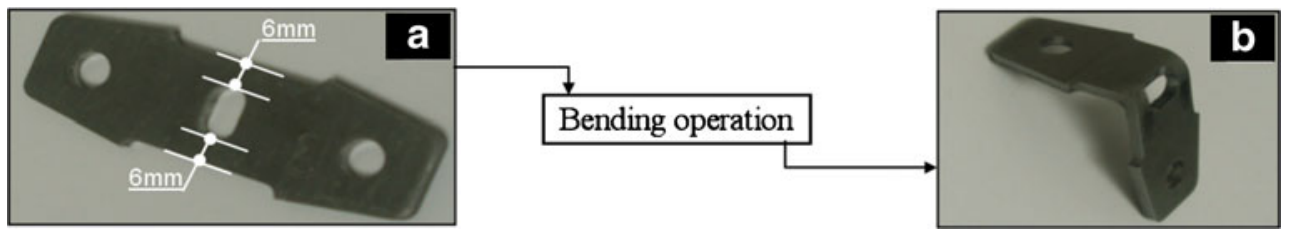


Table 2 Experimental design matrix of maximum bending load $(K N)$

\begin{tabular}{llllllll}
\hline$R_{\mathrm{d}}(\mathrm{mm})$ & \multicolumn{7}{l}{ Clearance $(\mathrm{mm})$} \\
\cline { 2 - 7 } & -0.6 & -0.4 & -0.2 & 0 & 0.2 & 0.4 & 0.6 \\
\hline 1 & 10.3 & 9.454 & 9.112 & 8.266 & 8.335 & 7.923 & 7.329 \\
2 & 9.18 & 9.294 & 8.464 & 7.542 & 7.908 & 6.836 & 6.43 \\
4 & 7.535 & 7.496 & 7.481 & 7.047 & 7.496 & 6.636 & 6.255 \\
6 & 7.565 & 7.344 & 7.169 & 6.932 & 6.841 & 6.856 & 6.673 \\
\hline
\end{tabular}

The second objective function represents the springback. The effects of these process parameters previously described on the variation of springback of S500MC steel sheet has been investigated in this study. As the springback phenomenon is caused by elastic recovery of deformed sheet, the control of elastic recovery is important in decreasing the amount of springback.

The process sequences of one of the tested specimens photographed during the wiping die bending experiments is shown in Fig. 3.

After having bent all the specimens, visual observations and measurements of the final angle are carried out by using a profile projector (type: $S A G E M-P 500-67 N^{\circ} 524$ ) in order to quantify the value of springback. Figure 4 shows an illustration of this instrument.

For this reason, a DoE with two parameters is constructed. It includes two factors which are the punch-sheet clearance and the die radius. They present the same discretisation like in the previous study of bending load. Each measurement of springback was repeated five times and, consequently, the mean value is calculated by the following relation: $\theta_{\text {mean }}=\frac{1}{5} \sum_{i=1}^{5} \theta_{\text {imeasured }}$.

The experimental design matrix representative of average values of this response for each combination of process parameters is reported in Table 3.

Figure 5a represents the possibility of negative clearance values which can be explained by a distance between punch and die lower than the workpiece thickness $t$. A smaller clearance defines a larger maximum strain at the edge of the sheet metal part, which decreases the relative influence of elastic deformation. For negative or zero clearances, the friction will be one of the causes of increase of loads allowing to a marked stress state especially at the interface between punch and blank. Figure 5b shows the state of bent specimens subjected to friction generated by punch. It corresponds to a bending test with die radius of $1 \mathrm{~mm}$ and negative clearance of $-0.6 \mathrm{~mm}$. Especially for specimens with an oblong hole, localisation zones of damage and cracks are detected at rounded-offs of oblong hole. The same phenomenon was observed for other die redii. On the marked zone, there will be significant reduction of the thickness of specimen and the appearance of cracks, as a result of punch-die clearance lower than the thickness.

\section{Procedures of optimization}

\subsection{Optimization problem}

In sheet metal bending processes, various criteria must be satisfied, and the search for an optimal solution can account for contradictory criteria. Consequently, several constraints and objective functions are necessary in order to obtain
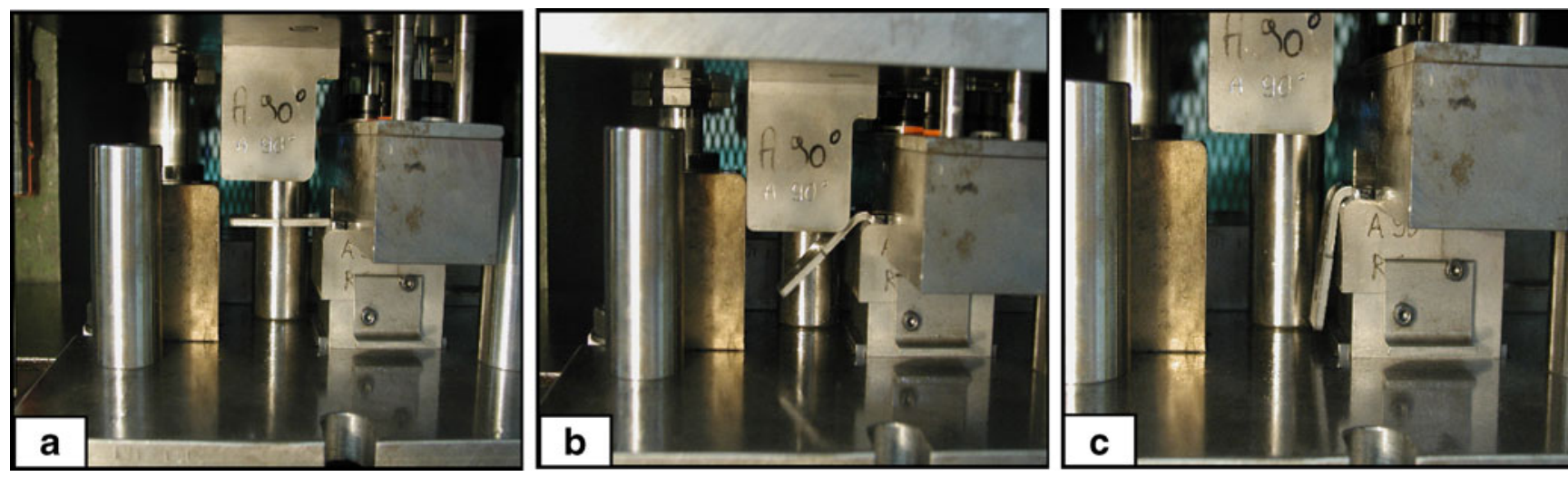

Fig. 3 The process sequence photographed during the wiping die bending experiments of high-strength low-alloyed steel (S500MC). (a) Initial position, (b) intermediate phase, and (c) springback 


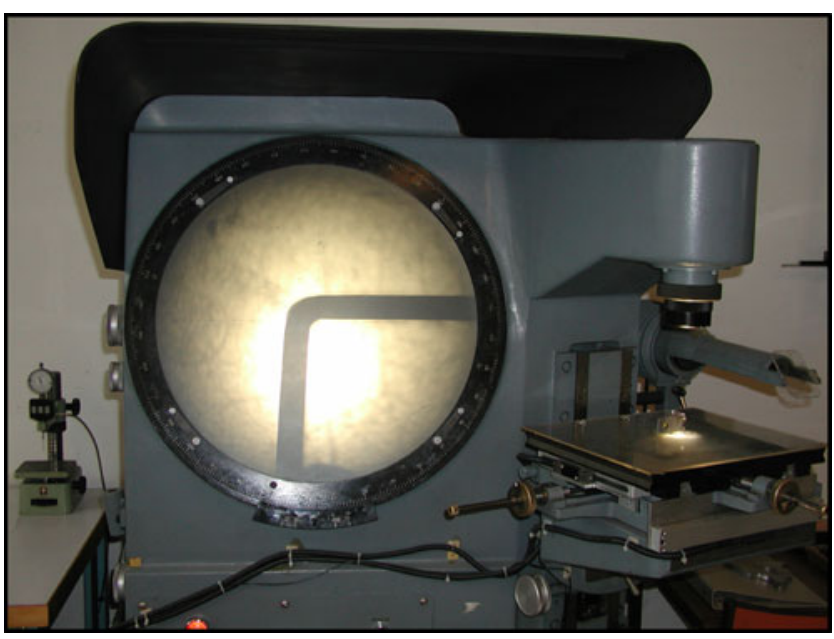

Fig. 4 Profile projector for measuring the springback angle. Model P500_67 ${ }^{\circ} 524$

proper quality product. The optimization problem can be stated as follows:

$(P):\left\{\begin{array}{l}\operatorname{Min} Y(\mathbf{x}) \\ \text { subject to } \\ g_{i}(\mathbf{x}) \geq 0 \quad 1 \leq i \leq r . \\ h_{i}(\mathbf{x})=0 \quad 1 \leq i \leq s \\ \text { with }: \mathbf{x}^{u} \leq \mathbf{x} \leq \mathbf{x}^{v}\end{array}\right.$

where $r$ and $s$ are, respectively, the number of inequalities, $g_{i}(\mathbf{x})$, and equalities, $h_{i}(\mathbf{x})$, constraints, $Y(\mathbf{x})$ is the objective function, and $\mathbf{x}^{\mathrm{u}}$ and $\mathbf{x}^{\mathrm{v}}$ are the lower- and upper-bound vectors of the design variable $\mathbf{x}$.

\subsection{Overview of response surface method}

Response surface modelling techniques were originally developed to analyse the results of physical experiments and to create an empirical model of the observed response values [39]. Response surface modelling postulates a model in the form of:

$\widetilde{Y}(\mathbf{x})=Y(\mathbf{x})+\varepsilon$

where $\tilde{Y}(\mathbf{x})$ is the approached function of $Y(\mathbf{x})$ at point $\mathbf{x}$, and $\varepsilon$ is the random error which has often an uniform distribution with a mean equal to zero and a variance $\sigma^{2}$.
The polynomial function, $\widetilde{Y}(\mathbf{x})$, used to approximate $Y(\mathbf{x})$ is typically a low order polynomial which is assumed to be either linear (Eq. 3), or quadratic (Eq. 4).

$\widetilde{Y}=\beta_{0}+\sum_{i=1}^{k} \beta_{i} x_{i}$

$\widetilde{Y}=\beta_{0}+\sum_{i=1}^{k} \beta_{i} x_{i}+\sum_{i=1}^{k} \beta_{i i} x_{i}^{2}+\sum_{i=1}^{k-1} \sum_{j>i}^{k} \beta_{i j} x_{i} x_{j}$

The parameters, $\beta_{0}, \beta_{i}, \beta_{i i}$, and $\beta_{i j}$ of the polynomials in Eqs. 3 and 4 are determined through least squares regression which minimises the sum of the squares of the deviations of predicted values, $\widetilde{Y}(\mathbf{x})$, from the actual values, $Y(\mathbf{x})$. The coefficients of Eqs. 3 and 4 used to fit the model can be found using least squares regression given by Eq. 5 :

$\beta=\left[X^{\prime} X\right]^{-1} X^{\prime} \mathbf{Y}$

where $\mathbf{X}$ is the design matrix of sample data points, $\mathbf{X}^{\prime}$ is its transpose, and $\mathbf{Y}$ is a column vector containing the values of the response at each sample point. For more details on least squares regression or polynomial response surface see, e.g., the reference of Myers and Montgomery [22].

3.3 Optimization procedure based on an SQP algorithm

Various methods can be used to approach the nonlinear optimization problem. Here, the MLS method [40, 41] is used to obtain an explicit expression of the objective function:

$\widetilde{Y}(\mathbf{x})=\sum_{i=1}^{m} p_{i}(\mathbf{x}) \cdot \beta_{i}(\mathbf{x})=\mathbf{p}^{\mathbf{T}}(\mathbf{x}) \cdot \beta(\mathbf{x})$

where $\mathbf{p}(\mathbf{x})=\left[p_{1}(\mathbf{x}), \ldots, p_{m}(\mathbf{x})\right]^{\mathrm{T}}$ is a finite set of $m$ polynomial basis functions, and $\boldsymbol{\beta}(\mathbf{x})=\left[\beta_{1}(\mathbf{x}), \ldots, \beta_{m}(\mathbf{x})\right]^{\mathrm{T}}$ are the unknown coefficients.
Table 3 Experimental design matrix of springback angle in (degrees)

\begin{tabular}{llllllll}
\hline$R_{\mathrm{d}}(\mathrm{mm})$ & \multicolumn{6}{l}{ Clearance $(\mathrm{mm})$} \\
\cline { 2 - 7 } & -0.6 & -0.4 & -0.2 & 0 & 0.2 & 0.4 & 0.6 \\
\hline 1 & 2.5 & 2.666 & 2.783 & 3 & 3.633 & 4.016 & 4.466 \\
2 & 2.966 & 2.95 & 3 & 3.25 & 3.866 & 4.366 & 4.833 \\
4 & 3.033 & 3.13 & 3.2 & 3.566 & 4.416 & 5.116 & 6.05 \\
6 & 3.2 & 3.216 & 3.216 & 3.916 & 4.8 & 5.8 & 6.65 \\
\hline
\end{tabular}


Fig. 5 a Possibility of bending operation with negative clearance $\mathrm{C}=-0.6 \mathrm{~mm}$ and (b) crack formation in the bending zone and marks of friction for $R_{\mathrm{d}}=$ $1 \mathrm{~mm}$ and $\mathrm{C}=-0.6 \mathrm{~mm}$
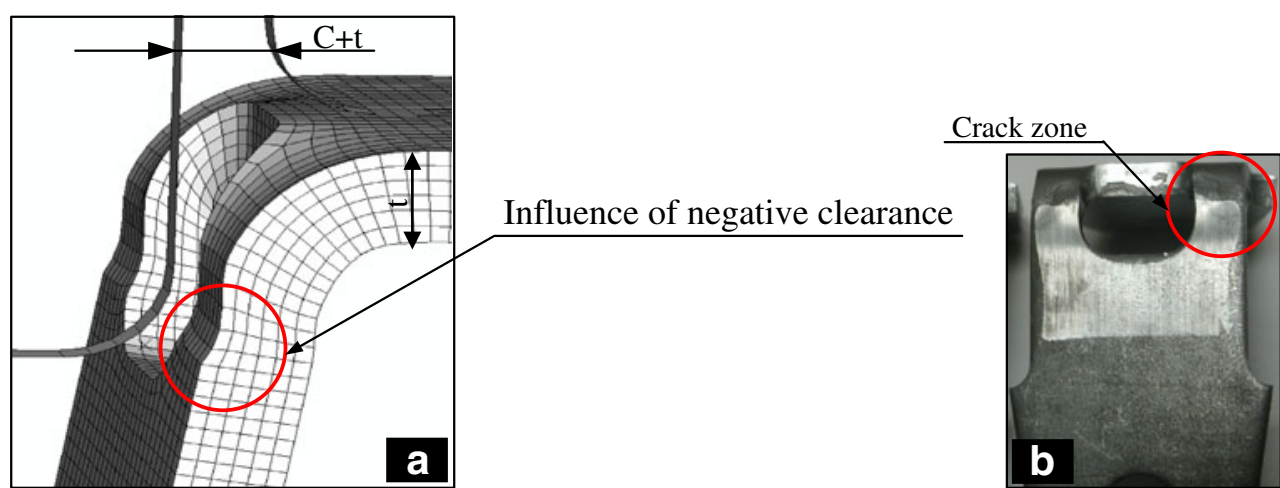

The MLS technique consists to determinate the coefficients $\beta$ allowing to minimise locally (in the vicinity of $\mathbf{x}$ ) the error, $R(\mathbf{x})$, between the exact and the approximated values of the function at the $N$ sampling points, $x_{I}$ :

$$
\begin{aligned}
R(\mathbf{x}) & =\sum_{I=1}^{N} w\left(\mathbf{x}-\mathbf{x}_{I}\right)\left(\widetilde{Y}\left(\mathbf{x}_{I}\right)-Y\left(\mathbf{x}_{I}\right)\right)^{2} \\
& =\sum_{I=1}^{N} w\left(\mathbf{x}-\mathbf{x}_{I}\right)\left(\mathbf{p}^{\mathbf{T}}\left(\mathbf{x}_{I}\right) \cdot \beta(\mathbf{x})-Y\left(\mathbf{x}_{I}\right)\right)^{2} \\
& =[\mathbf{P} \cdot \beta(\mathbf{x})-\mathbf{Y}]^{\mathrm{T}} \cdot \mathbf{W} \cdot[\mathbf{P} \cdot \beta(\mathbf{x})-\mathbf{Y}]
\end{aligned}
$$

The matrices $\mathbf{Y}, \mathbf{P}$, and $\mathbf{W}$ are defined as:

$$
\mathbf{Y}^{\mathrm{T}}=\left\langle Y\left(\mathbf{x}_{1}\right), Y\left(\mathbf{x}_{2}\right), \ldots, Y\left(\mathbf{x}_{N}\right)\right\rangle
$$

$\mathbf{P}=\left[\begin{array}{c}\mathbf{p}^{\mathrm{T}}\left(\mathbf{x}_{1}\right) \\ \mathbf{p}^{\mathrm{T}}\left(\mathbf{x}_{2}\right) \\ \vdots \\ \mathbf{p}^{\mathrm{T}}\left(\mathbf{x}_{N}\right)\end{array}\right]_{N \times m}$

$\mathbf{W}(\mathbf{x})=\left[\begin{array}{cccc}w\left(\mathbf{x}-\mathbf{x}_{1}\right) & 0 & \ldots & 0 \\ 0 & w\left(\mathbf{x}-\mathbf{x}_{2}\right) & \ldots & 0 \\ \vdots & \vdots & \vdots & \vdots \\ 0 & 0 & \ldots & w\left(\mathbf{x}-\mathbf{x}_{N}\right)\end{array}\right]_{N \times N}$

where $\left(\mathbf{x}_{I}\right)_{I=1,2, \ldots, N}$ are the $N$ evaluation points in the DoE, $w$ $\left(\mathbf{x}-\mathbf{x}_{I}\right)$ is the weight function used in the MLS fitting which can take different forms as shown in some papers [10]. The following weight function proposed by Häussler-Combe and Korn at 1998 [42] is used:

$w\left(\mathbf{x}-\mathbf{x}_{I}\right)= \begin{cases}\left(\mathrm{e}^{-\left(\frac{\left\|\mathbf{x}-\mathbf{x}_{I}\right\|}{\alpha \cdot d}\right)^{2}}-\mathrm{e}^{-\frac{1}{\alpha^{2}}}\right) /\left(1-\mathrm{e}^{-\frac{1}{\alpha^{2}}}\right) & \text { if }\left\|\mathbf{x}-\mathbf{x}_{I}\right\| \leq d \\ 0 & \text { else } .\end{cases}$
This function $w\left(\mathbf{x}-\mathbf{x}_{I}\right)$ is positive and its value decreases with the distance, $\left\|\mathbf{x}-\mathbf{x}_{I}\right\|$, between the sampling point $\mathbf{x}$ and the node $\mathbf{x}_{I}$. It always takes the unit value at the sampling point $\left(\mathbf{x}=\mathbf{x}_{I}\right)$ and zeros value outside the influence domain, which is defined by the value, $d$.

The parameter $\alpha$ allows to control the weight function curve in the domain of influence [41, 42]. When the value of $\alpha$ decreases, the influence domain in the MLS technique will be more concentrated around the region near the fitting point, $\mathbf{x}$. The parameter $\alpha$ is fixed to $1 / 3$.

The minimization of $R(\mathbf{x})$ in Eq. 7 compared to $\beta_{i}(\mathbf{x})$ leads to the following linear relation between $\boldsymbol{\beta}(\mathbf{x})$ and $\mathbf{Y}$ :

$\frac{\partial \mathbf{R}}{\partial \boldsymbol{\beta}}=0$

$\mathbf{A}(\mathbf{x}) \cdot \boldsymbol{\beta}(\mathbf{x})=\mathbf{B}(\mathbf{x}) \cdot \mathbf{Y}$

where the matrices $\mathbf{A}(\mathbf{x})$ and $\mathbf{B}(\mathbf{x})$ are defined by:

$$
\begin{aligned}
\mathbf{A}(\mathbf{x}) & =\mathbf{P}^{\mathbf{T}} \mathbf{W}(\mathbf{x}) \mathbf{P} \\
& =\sum_{I=1}^{N} w\left(x-x_{I}\right) P\left(x_{I}\right) P^{T}\left(x_{I}\right)
\end{aligned}
$$

$$
\begin{aligned}
\mathbf{B}(\mathbf{x}) & =\mathbf{P}^{\mathbf{T}} \mathbf{W}(\mathbf{x}) \\
& =\left\langle w\left(x-x_{1}\right) P\left(x_{1}\right), w\left(x-x_{2}\right) P\left(x_{2}\right), \ldots, w\left(x-x_{N}\right) P\left(x_{N}\right)\right\rangle
\end{aligned}
$$

The unknown coefficients $\beta(\mathbf{x})$ can be achieved by solving Eq. $12 \mathrm{a}$ and $12 \mathrm{~b}$, which results in:

$\beta(\mathbf{x})=\mathbf{A}^{-1}(\mathbf{x}) \mathbf{B}(\mathbf{x}) \mathbf{Y}$ 
Substituting the unknown coefficient from Eq. 15 into Eq. 6 leads to the MLS approximation of the estimated value $\widetilde{Y}(\mathbf{x})$ as:

$\widetilde{Y}(\mathbf{x})=\mathbf{p}^{T}(\mathbf{x}) \mathbf{A}^{-1}(\mathbf{x}) \mathbf{B}(\mathbf{x}) \mathbf{Y}$

\subsubsection{Optimization strategy}

In order to reach the global optimum with precision, an optimization procedure based on an SQP algorithm and a strategy of actualization of the response surface is used. It means that at the next iteration, the computations are performed as previously for the new points, but the response surface is fitted now using the SQP approach over all current and previous experimental design. Global and local approximations are built.

Since the MLS approximation is accurate locally, it's possible that the SQP algorithm finds a local optimum. This is due to the presence of several local minima towards which the region of interest converges. They show that the response surface is not convex, and the search of the global optimum value should be carried out with several initial points located inside the feasibility domain. To avoid this difficulty, a global approximation is made initially. The weight functions are equal to one. After that, successive local approximations are built, in the vicinity of the optima $\mathbf{x}_{\text {opt }}^{k}$. In the SQP method, the convergence properties depend on the starting point. The sequence $\mathbf{x}$ converges quadratically to the optimal solution $\mathbf{x}_{\mathrm{opt}}^{k}$, if the starting values $\mathbf{x}^{0}$ are sufficiently close to the minimum point $\mathbf{x}_{\mathrm{opt}}^{k}$. The method may fail, e.g., in situations where the starting point is not close enough to the minimum.
During the progression of the procedure, the region of interest moves and zooms on each optimum, $\mathbf{x}_{\mathrm{opt}}^{k}$ (Fig. 6). The influence region is more and more concentrated around the optimum, $\mathbf{x}_{\mathrm{opt}}^{k}$, by decreasing the diameter, $d$, in the following way:

$d_{k+1}=\left\{\begin{array}{lc}d_{\min } & \text { if } d_{k}\left|\left(\frac{k}{k+1}\right)^{2}-1\right| \leq d_{\min } \\ d_{k}\left|\left(\frac{k}{k+1}\right)^{2}-1\right| & \text { else }\end{array}\right.$.

where $d_{\min }$ is a minimal diameter to be respected.The number of the discrete points in the interest region is determined by the size of the influence domain, $d$, and it must be always higher than the unknown coefficients number. Since the MLS approximation is precise only in the vicinity of $\mathbf{x}$, the lower and upper bounds of each design variable, $x_{i}$, are limited (Fig. 6) at each approximation as follows:

$x_{i}^{u}=\left\{\begin{array}{cc}x_{i}-d & \text { if } x_{i}-d>x_{i}^{\min } \\ x_{i}^{\min } & \text { else }\end{array}\right.$

$x_{i}^{v}=\left\{\begin{array}{cc}x_{i}+d & \text { if } x_{i}+d<x_{i}^{\max } \\ x_{i}^{\max } & \text { else }\end{array}\right.$

where the scalars $x_{i}^{u}$ and $x_{i}^{v}$ are the actualized lower and upper bounds of the $i$ th design variable, $x_{i}$, and $x_{i}^{\min }$ and $x_{i}^{\max }$ are the lower and upper bounds of the DoE.The optimization procedure is stopped when the number of approximations exceeds ten or when the optima of the last two approximations are very close (Eq. 20):

$\left\|\mathbf{x}_{\text {opt }}^{k+1}-\mathbf{x}_{\text {opt }}^{k}\right\| \leq 10^{-4}$
Fig. 6 Progression of the optimization procedure for $2 \mathrm{D}$ case: global (a) and successive local (b) approximations

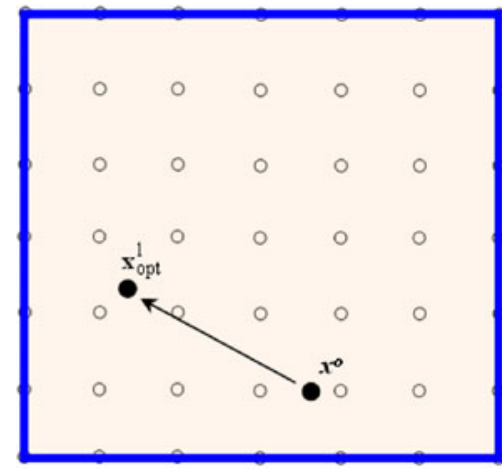

(a)

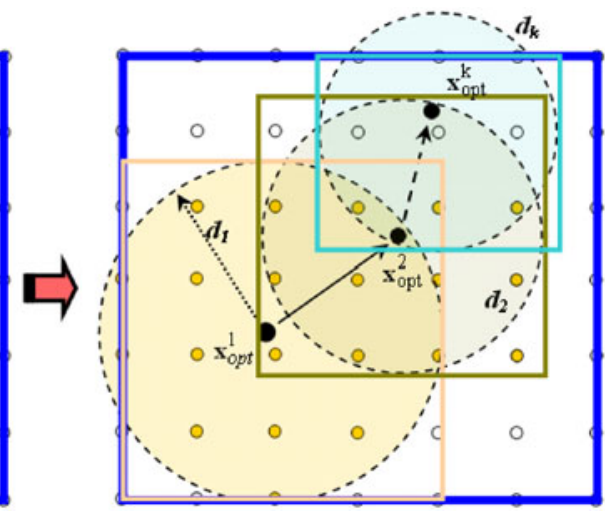

(b)

Validity domain of the initial global approximation Validity domain of the first local approximation

Validity domain of the second local approximation

- Validity domain of the kth local approximation 
The goal of this procedure is to improve the precision of the RSM in the vicinity of the optimum and to lead the optimization algorithm to the global optimum. The methodology is shown in Fig. 7.

\subsection{Optimization procedure based on the evolutionary algorithms}

Evolutionary algorithms are a set of computer procedures of search and optimization based on the concept of the mechanism of natural selection and genetics. They are well adapted when the functions are noisy, discontinuous, nondifferentiables, and when they have numerous local optima. They can be classified in evolutionary programming, ES, and genetic algorithms (GA). The essential difference between the ES and the GA is the adaptation of the mutation distribution. In the both cases, the same operators are used: selection, mutation, and cross-over operators. A summary of features and applications of ES in design optimization has been given [5]. It shows that the specialised variants of ES are interesting algorithms for tackling complex inverse design problems in computational mechanics, because they provide a high robustness, are sufficiently fast, simple to apply, and easy to run. In ES, archived information of all previous evaluations can be used in order to place new search points and to avoid the exploration of less promising and already explored regions of the search space. Consequently, this leads to a very powerful technique for global and local optimization problems and to significantly better results.

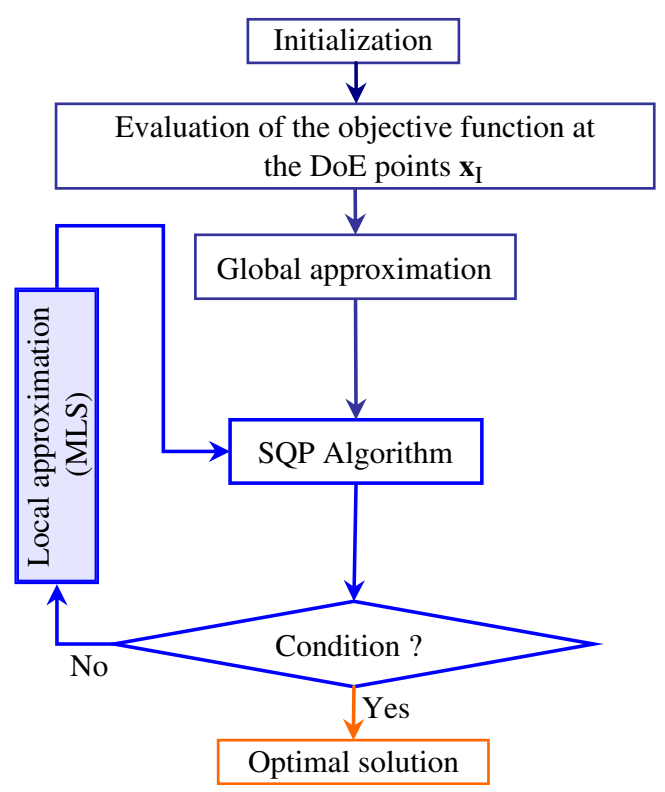

Fig. 7 Flow chart of optimization procedure based on sequential quadratic programming algorithm

\subsubsection{Evolution strategies}

In ES, a population of individuals is created with $\mu$ parents and $\lambda$ children. Each individual is represented by a vector $\mathbf{X}$ with $n$ real components $x_{k}$ representing its genotype (X€ $\mathfrak{R}^{n}$ ), and the population evolves according to the operations of selection, mutation, and cross-over.

Mutation in a binary coding scheme involves switching individual bits along the string, changing a zero to one or vice versa. This operator keeps the diversity of the population and reduces the possibility that the ES find a local minimum or maximum instead of the global solution, although this is not ever guaranteed. In the step of mutation, the strategy generates an offspring (new children), in which the capacity of survival is given by calculating the values of the objective function. The offspring represented by the vector $\mathrm{X}_{\mathrm{N}}^{(g)}$ (index $\mathrm{N}$ for new), is obtained by adding to the parent $\mathrm{X}_{O}^{(g)}$ (index $\mathrm{O}$ for old) a random vector $\mathbf{Z}$ with normally distributed components $\mathbf{Z} \sim \mathrm{N}(\mathbf{0}, \mathbf{1})$ with $\mathbf{Z} \in \mathfrak{R}^{n}$ :

$\mathrm{X}_{N}^{(g)}=X_{O}^{(g)}+\sigma^{(g)} \mathrm{Z}$

$\sigma^{(g)}$ is the global step size determining the length of the mutation step in generation $(\mathrm{g})\left(\sigma \in \mathfrak{R}_{>0}\right)$.

In the step of selection, at the same time for the parent and the offspring, the capacity of survival corresponding to the objective function evaluation is calculated. In a context of minimization, the individual with the smallest value is selected to be the parent in the next generation.

The case of a strategy with one parent and one offspring can be described as follows:

1. Design of an offspring:

$\mathrm{X}_{N}^{(g)}=X_{O}^{(g)}+\sigma^{(g)} \mathrm{Z}$

2. Selection:

$\mathrm{X}_{O}^{(g+1)}= \begin{cases}X_{N}^{(g)} & \text { if } f\left(X_{N}^{(g)}\right) \leq f\left(X_{O}^{(g)}\right) \\ X_{O}^{(g)} & \text { otherwise. }\end{cases}$

3. If the loop is not finished, make $\mathrm{g}=\mathrm{g}+1$ and go to $\mathbf{i}$.

In case of ES for several individuals, the operator for recombination, that is the cross-over between individuals, is generally employed before mutation. The recombination 
operator allows interchanging of the components $x_{k}^{\text {par1 }}$ and $x_{j}^{\text {par2 }}$ of the two vectors constituting the parents par1 and par2. Genes of several parents can also be mixed by a multiple operation of recombination. In the case of two parents, $\mathbf{X}^{1}$ and $\mathbf{X}^{2}$, an offspring $\mathbf{X}^{\prime}$ can be obtained by arithmetic recombination of the components according to the following way:

$x_{i}^{\prime}=a x_{i}^{1}+(1-\alpha) x_{i}^{2}$

$\alpha$ is a parameter $\in[0,1]$ and $i=1,2, \ldots, n . n$ is the number of genes in the individuals. If the number of parents is $\mu>2$, Eq. 21 is an intermediate recombination.

In ES, the choice of strategic parameters like the population size, the size of mutation step, or the selection scheme is crucial for the strategy behaviour and convergence properties. A detailed attention must be paid to the adaptation of the step size during the course of optimization.

\subsubsection{Algorithm of the CMA-ES}

Following Hansen and Ostermeier [33], in the $\left(\mu_{I}, \lambda\right)$ derandomized CMA-ES, the $\lambda$ offspring of generation $\mathrm{g}+$ 1 are computed by:

$x_{k}^{(g+1)}=\langle x\rangle_{\mu}^{(g)}+\underbrace{\sigma^{(g)} \mathrm{B}^{(g)} D^{(g)} z_{k}^{(g+1)}}_{\sim N\left(0, C^{(g)}\right)}, \quad k=1, \ldots, \lambda$,

where

$\langle x\rangle_{\mu}^{(g)}=\frac{1}{\mu} \sum_{i \in I_{s e l}^{(\mathrm{g})}} x_{i}^{(g)}$

represents the centre of mass of the selected individuals of generation $\mathrm{g}$, and $I_{\text {sel }}^{(\mathrm{g})}$ is the set of indices of the selected indivduals of generation $\mathrm{g}$ with $\left|I_{\text {sel }}^{(g)}\right|=\mu \cdot \sigma^{(g)}$ is the global step size.

The random vectors $z_{k}$ from Eq. 25 are $N(\mathbf{0}, \mathbf{I})$ distributed (n-dimensional normally distributed with expectation zero and the identity covariance matrix) and serve to geneate offspring for generation $\mathrm{g}+1$. Similar to Eq. 26, we can calculate their centre of mass as:

$\langle z\rangle_{\mu}^{(g+1)}=\frac{1}{\mu} \sum_{i \in I_{\mathrm{sel}}^{(\mathrm{g}+1)}} z_{i}^{(g+1)}$

The covariance matrix $\mathbf{C}^{(\mathrm{g})}$ of the random vectors $\mathbf{B}^{(\mathrm{g})}$, $\mathbf{D}^{(\mathrm{g})}$, and $z_{k}^{(g+1)}$ is a symmetrical positive $n \times n$ matrix. The columns of the orthogonal matrix $\mathbf{B}^{(\mathrm{g})}$ represent normalised eigenvectors of the covariance matrix. $\mathbf{D}^{(\mathrm{g})}$ is a diagonal matrix whose elements are the square roots of the eigenvalues of $\mathbf{C}^{(\mathrm{g})}$. Hence, the relation of $\mathbf{B}^{(\mathrm{g})}$ and $\mathbf{D}^{(\mathrm{g})}$ to $\mathbf{C}^{(\mathrm{g})}$ can be expressed by:

$C^{(g)}=\mathrm{B}^{(g)} D^{(g)}\left(\mathrm{B}^{(g)} D^{(g)}\right)^{T}$ and $C^{(g)} b_{i}^{(g)}=\left(d_{i i}^{(g)}\right)^{2} \cdot b_{i}^{(g)}$

where $b_{i}^{(g)}$ represents the $i$ th column of $\mathbf{B}^{(\mathrm{g})}$ and $\left\|b_{i}^{(g)}\right\|=1$ and $d_{i i}^{(g)}$ are the diagonal elements of $\mathbf{D}^{(\mathrm{g})}$. Surfaces of equal probability density of the random vector $B^{(g)} D^{(g)} z_{k}^{(g+1)} \sim$ $N\left(0, C^{(g)}\right)$ are (hyper-)ellipsoids whose main axes correspond to the eigenvectors of the covariance matrix. The squared lengths of the axes are equal to eigenvalues of the covariance matrix.

In the following, the two adaptation mechanism of the CMA-ES are described: (1) the adaptation of the covariance matrix $\mathbf{C}^{(\mathrm{g})}$ and (2) the adaptation of the global step size $\sigma^{(\mathrm{g})}$.

First, the evolution path $p_{c}^{(g+1)}$ is calculated by:

$$
\begin{aligned}
p_{c}^{(g+1)}= & \left(1-c_{\mathrm{c}}\right) \cdot p_{c}^{(g)} \\
& +\sqrt{c_{c} \cdot\left(2-c_{c}\right)} \cdot \underbrace{\frac{\sqrt{\mu}}{\sigma^{(g)}}\left(\langle x\rangle_{\mu}^{(g+1)}-\langle x\rangle_{\mu}^{(g)}\right)}_{=\sqrt{\mu} B^{(g)} D^{(g)}\langle z\rangle_{\mu}^{(g+1)}}
\end{aligned}
$$

it is used to build the covariance matrix of generation $\mathrm{g}+1$.

$C^{(g+1)}=\left(1-c_{c o v}\right) \cdot C^{(g)}+c_{c o v} \cdot p_{c}^{(g+1)}\left(p_{c}^{(g+1)}\right)^{\mathrm{T}}$

$\mathbf{C}$ is updated with a symmetric matrix of rank one (right summand in Eq. 30). Note here that using $c_{c}=1$ in Eq. 29 reduces the evolution path to $\sqrt{\mu} B D\langle z\rangle_{\mu}$ which is the mean mutation step of the last generation multiplied by $\sqrt{\mu}$.

Choosing $c_{c}<1$ makes the adaptation usually faster and more reliable as the correlation between successive steps is exploited [33].

Second, to adapt the global step size $\sigma$, the evolution path $p_{\sigma}^{(g+1)}$ is computed in anology to the evolution path $\mathbf{p}^{(\mathrm{g}+1)}$. The difference between the two evolution paths is that $p_{\sigma}^{(g+1)}$ is not scaled by $\mathbf{D}^{(\mathrm{g})}$, resulting in:

$$
\begin{aligned}
p_{\sigma}^{(g+1)}= & \left(1-c_{\sigma}\right) \cdot p_{\sigma}^{(g)} \\
& +\sqrt{c_{\sigma} \cdot\left(2-c_{\sigma}\right)} . \underbrace{\sqrt{\mu} B^{(g)}\langle z\rangle_{\mu}^{(g+1)}}_{=B^{(g)}\left(D^{(g)}\right)^{-1}\left(B^{(g)}\right)^{-1} \frac{\sqrt{\mu}}{\sigma^{(g)}}\left(\langle x\rangle_{\mu}^{(g+1)}-\langle x\rangle_{\mu}^{(g)}\right)}
\end{aligned}
$$

The length of the evolution path determines the step size for generation $\mathrm{g}+1$,

$\sigma^{g+1}=\sigma^{(g)} \cdot \exp \left(\frac{1}{d_{\sigma}} \frac{\left\|\mathrm{P}_{\sigma}^{(g+1)}-\widehat{\chi}_{n}\right\|}{\widehat{\chi}_{n}}\right)$

where $\widehat{\chi}_{n}=\mathrm{E}[\|N(0, \mathrm{I})\|]$ is the expected length of a $(\mathbf{0}, \mathbf{I})$ normally distributed random vector, and $d_{\sigma}>1$ is the 
damping parameter. $\widehat{\chi}_{n}$ is approximated by $\widehat{\chi}_{n} \approx \sqrt{n} \times$ $\left(1-\frac{1}{4 n}+\frac{1}{21 n^{2}}\right)$ [34].

Equation 31 reveals that the length of the axes of the mutation ellipsoid does not affect the global step size adaptation.

The strategy parameter and setting is discussed by Hansen and Ostermeier [34] in detail, and the default setting is used as follows:

$$
\begin{aligned}
c_{c} & =\frac{4}{n+4}, c_{c o v}=\frac{2}{(n+\sqrt{2})^{2}}, c_{\sigma}=\frac{4}{(n+4)}, d_{\sigma} \\
& =c_{\sigma}^{-1}+1
\end{aligned}
$$

where the previous terms are defined by [33]:

$\left.\mathrm{c}_{\mathrm{c}} \in\right] \mathbf{0 , 1 ]}$ (cumulation for distribution): determines the cumulation time for $\mathbf{p}_{\mathrm{c}}$, which is roughly $1 / \mathrm{c}_{\mathrm{c}}$.

$\mathrm{c}_{\mathrm{cov}} \in \mathbf{0} \mathbf{0 , 1}$ (change rate of the covariance matrix $\mathbf{C}$ ): for $\mathrm{c}_{\mathrm{cov}}=0$, no change takes place.

$\mathbf{c}_{\sigma} \in \mathbf{0} \mathbf{0 , 1}$ (cumulation for step size): determines the cumulation time for $p_{\sigma}^{(g)}$, which is roughly $1 / \mathrm{c}_{\mathrm{c}}$

$d_{\sigma} \geq 1$ (damping parameter for step size): determines the possible change rate of $\sigma^{(\mathrm{g})}$ in the genration sequence.

Initial values are $\mathbf{P}^{(0)}=0, \mathbf{P}_{\sigma}^{(0)}=0$, and the intial covariance matrix $\mathbf{C}^{(0)}$ is the identity matrix $\mathbf{I}$.

\section{Optimization procedure based on the mathematics of response surface modelling}

The characteristic functions which were selected to approximate the responses of the parts during the wiping die bending operation are the maximum bending load and the springback. These last represent the objective functions of the optimization problem. Numerical and graphical optimization methods were used in this part of work by choosing the desired goals for each factor and response. For that, the RSM is used leading to a global representation of approximated functions at various sampled points of design space. Therefore, a MATLAB [43] based programs were developed in this work for which all computations were carried out.

The variables retained in this study, $C$ and $R_{\mathrm{d}}$, are reported to the sheet thickness $t$ such that the adimensional variables are defined as:

$\bar{C}=\frac{C}{t}$ and $\bar{R}_{\mathrm{d}}=\frac{R_{\mathrm{d}}}{t}$

For each measured response, a normalisation is carried out allowing to make easier the interpretation of the results and which permits the generalisation of the method for all type of variables. In all what follows, the maximum bending load $F_{\max }^{\text {bending }}$, and the springback $\theta$ are normalised to the yield force $F_{\mathrm{y}}$ and to the desired bending angle $\left(\alpha=90^{\circ}\right)$, respectively, in such a manner than the relative values of the responses can be written by the following ratios:

$\bar{F}=F_{\max }^{\text {bending }} / F_{y}$

$\bar{\theta}(\%)=\left(\frac{\theta-90}{90}\right) \times 100$

where $F_{\mathrm{y}}$ is the yield force calculated by multiplying the area of the initial section of the part $S$ by the yield stress $\sigma_{\mathrm{y}}$ of the used material.

$F_{y}=S \times \sigma_{y}$

Polynomial regression methods are commonly used to create response surface functions from a set of sampled data [44]. The general form of the approximate functions can be given by the following relation:

$\widehat{Y}(X)=F\left(x_{1}, x_{2}, x_{3}, \ldots, x_{n}\right)+\varepsilon$

where $\widehat{Y}(X)$ is the estimate of the target function at a point in the parameter space having $n$ independent coordinates $X=\left(x_{1}, x_{2}, x_{3}, \ldots, x_{n}\right) . \varepsilon$ represents other sources of variability not accounted for in $\widehat{Y}$. It includes effects such as experimental measurement error or numerical convergence error. In this study, we adopted a quadratic polynomial approximations in view to represent two models for the objective functions of maximum relative bending load and relative springback. Both can be formulated in the form defined by Eq. 4 .

Optimization is carried out in the search range of die radius and punch-sheet clearance in order to determine the optimal values of these process parameters guaranting the minimization of bending load and springback. The multiobjective function can be defined as follows:

Minimize $\bar{F}=f_{1}\left(\bar{C}, \bar{R}_{\mathrm{d}}\right)$

Minimize $\bar{\theta}=f_{2}\left(\bar{C}, \bar{R}_{\mathrm{d}}\right)$

Subject to

$\bar{R}_{\mathrm{d}_{\min }} \leq \bar{R}_{\mathrm{d}} \leq \bar{R}_{\mathrm{d}_{\max }}$

$\bar{C}_{\min } \leq \bar{C} \leq \bar{C}_{\max }$ 
The adequate final mathematical models in terms of listed factors are given below:

$$
\begin{aligned}
\bar{F}= & 0.356-0.147 \bar{R}_{\mathrm{d}}-0.419 \bar{C}+0.056 \bar{R}_{\mathrm{d}}^{2} \\
& -0.0545 \bar{C}^{2}+0.221 \bar{R}_{\mathrm{d}} \bar{C}
\end{aligned}
$$

$$
\begin{aligned}
\bar{\theta}= & 2.933+1.595 \bar{R}_{\mathrm{d}}+5.596 \bar{C}-0.352 \bar{R}_{\mathrm{d}}^{2} \\
& +35.476 \bar{C}^{2}+5.343 \bar{R}_{\mathrm{d}} \bar{C}
\end{aligned}
$$

4.1 The global evolution of maximum bending load and springback

The theoretical developments based on the realisation of experimental design make possible to determine analytical functions indicating the global representation of the maximum bending load. In order to identify the optimal values of the process parameters which are the punch-sheet clearance and the die radius, we chose to represent the evolution of the relative bending load given by Fig. 8. This mechanical response is normalised to the yield force $F_{\mathrm{y}}$ characterised by tensile tests.

The response surface illustrated by Fig. 8a is approximated by a mathematical model of second order. All interactions between the two considered parameters were taken into account. A decrease in the punch load which is more or less linear according to the clearance was noted. Regarding the trend of the response surface, it can also be noted that the maximum bending load decreases with the increase in the value of bending radius. The small values correspond to a positive clearances, and for a smallest radius, the force is hardly higher than for the largest radius.

Another remark concerns the curvature of the response surface which depends more strongly on the die radius than the clearance, which shows that $R_{\mathrm{d}}$ is the most sensitive parameter in this operation of sheet metal forming. The maximum values of the bending load are obtained at the most severe conditions characterised by small die radii and negative clearances which are considered as a forming punch, creating a bottoming at the top of the bend. This is explained essentially by the highest bending moment causing an increased strain hardening. The contour plots of the response surface are presented in Fig. 8b. They make possible to locate the optimal zone in which the maximum bending force is the smallest.

In order to better understand the evolution of springback, we chose the relative variation of the bending angle $\bar{\theta}(\%)=\left(\frac{\theta-90}{90}\right) \times 100$ compared to the mesured angle as an objective function. Here, it has been noted that the second order polynomial approximation was shown to be reliable and sufficient to fit the experimental data and to represent the global evolution of springback versus process parameters varying in the intervals $\bar{R}_{\mathrm{d}} \in[0.25,1.5]$ and $\bar{C} \in[-0.15,0.15]$. The correlation coefficients of the polynomial approximations of the maximum bending load and the springback objective functions are $98 \%$ and $95 \%$, respectively.

The results of this response are represented in Fig. 9. It can be observed that the maximum springback is reached for large values of clearance and die radius. The maximum value is about $7.38 \%$ of the bending angle and which is reached for $\bar{R}_{\mathrm{d}}=1.5$ and $\bar{C}=0.15$. Minimum springback is obtained for the most severe conditions given by the smallest die radii and negative clearances. In this case, significant shearing occurs between punch and sheet, which leads to a decrease in the resistance of the material. This results in a decrease in the relative springback angle.

In the negative domain of clearances, the variation of springback does not seem to be very sensitive to the variation of die radii. Whereas, the springback increases more rapidly in the positive domain of relative clearances $\bar{C}$.
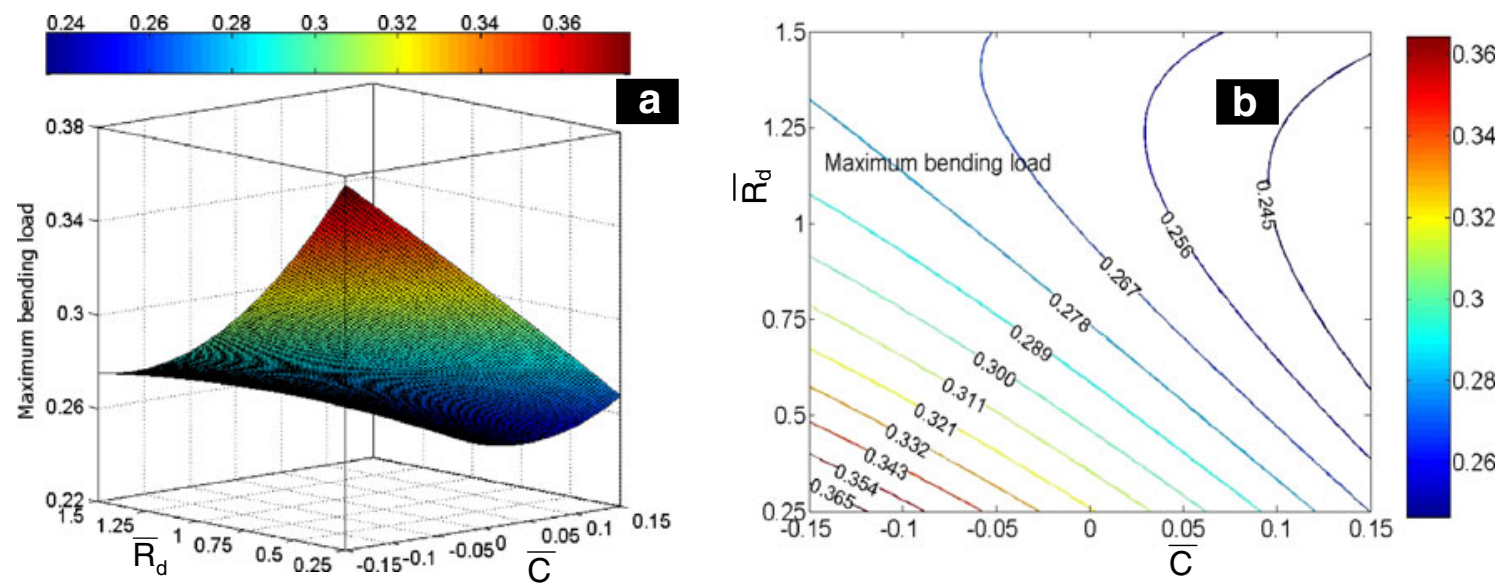

Fig. 8 Second order response surface (a) and contour plots (b) of two variables for maximum bending load function 

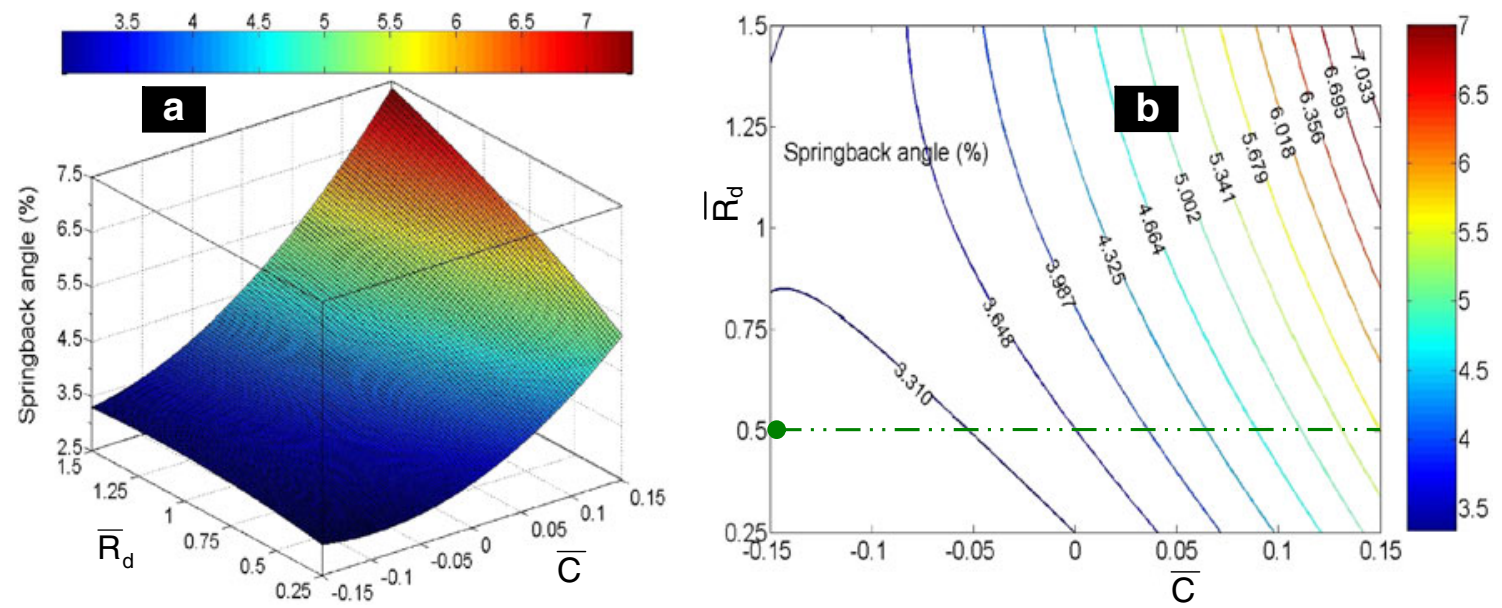

Fig. 9 Second order response surface (a) and contour plots (b) of two variables for springback function

Figure 9a is a three-dimensional representation of the relative variation of measured springback given in the form of response surface. The result shows that the springback evolves in a nonlinear way according to the considered parameters and that it is more sensitive to the clearance than to the die radius. The slope of springback variation with die radius increases with the increasing of relative clearance as shown in Fig. 9a. From a practical point of view, it should be noted that the net-shape of parts can not be obtained easily with good geometrical properties in wiping die bending process for positive relative punch-sheet clearance. Consequently, it is recommended to bend the parts by adopting small clearance. The variation of springback in the space design is illutrated by contour plots shown in Fig. 9b allowing to locate the regions corresponding to the minimum springback. This one is obtained in the lower left zone of Fig. $9 \mathrm{~b}$ found for small values of clearance and die radius. It was limited by amplitude lower than $3.31 \%$. However, it would be necessary to limit the useful zone at $\bar{R}_{\mathrm{d}}=0.5$ below which nonremediable cracks appear (see dotted line in Fig. 9b). In this reference, the springback is attained at limits $\bar{R}_{\mathrm{d}}=0.5$ and $\bar{C}=-0.15$ and its value is equal to $2.88^{\circ}$.

\section{Optimization results and comparison}

\subsection{Results of optimization by global approach}

The purpose of the study is to determine the optimal relative values of process parameters which make possible to minimise bending load and springback. In order to do this, the two response surfaces corresponding to the two objectives functions are plotted. The optima in the global approach are found by evaluating the values of the objectives functions at points located inside the feasibility domain. The latter is sampled by a regular grid of $100 \times 100$ points which corresponds to a discretisation of each process parameters. Figure 10 shows the flow chart of the optimization step.

The minimum value of bending load is $\bar{F}_{\text {min }}^{\text {Global }}=0.2348$. This global minimum is reached when $\bar{R}_{\mathrm{d}}=1$ and $\bar{C}=0.15$. This approach is applied again for the minimization of springback objective function. Hence, the optimal solution is obtained for a minimum springback value equal to $\bar{\theta}_{\text {min }}^{\text {Global }}=2.9715$ corresponding to the values of geometrical parameters of the process $\bar{R}_{\mathrm{d}}=0.25$ and $\bar{C}=-0.099$. The CPU time for each simulation is approximately $5 \mathrm{~s}$.

\subsection{Optimization results obtained using an SQP algorithm}

Here, we propose to apply an optimization technique based on the SQP algorithm in view to determine the optimal values of die radius and punch-sheet clearance which optimise the maximum bending load and springback. The

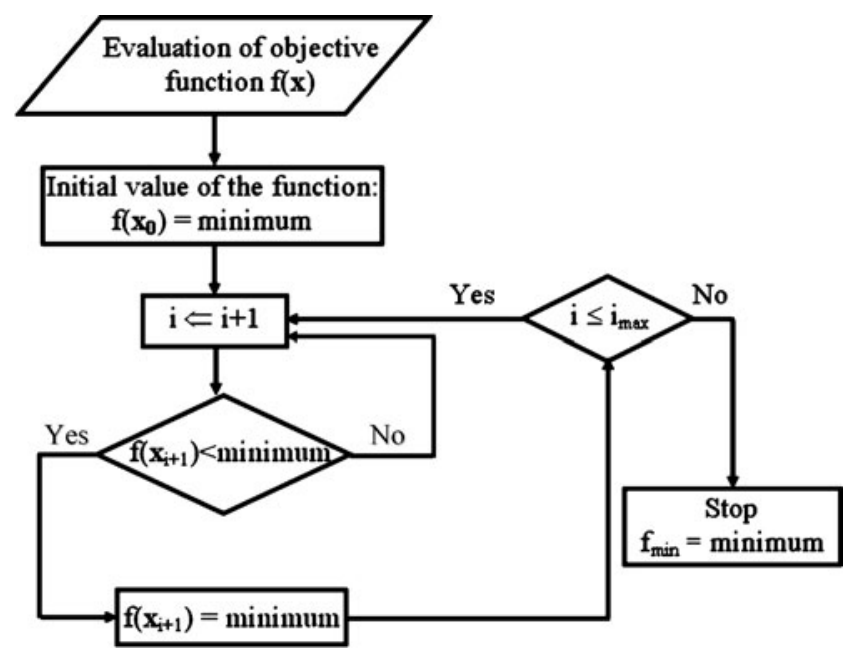

Fig. 10 Flow chart of global optimization algorithm 


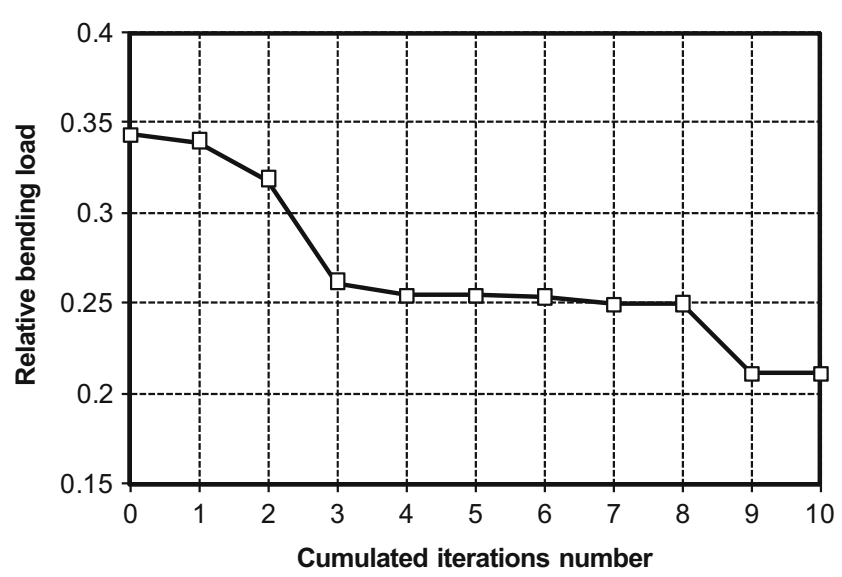

Fig. 11 Evolution of relative bending load versus iterations number

average calculation time for solving the two optimization problems was about $25 \mathrm{~s}$.

\subsubsection{Minimization of the maximum bending load}

The minimization of objective function versus the iterations is represented by Fig. 11. For this simulation, the coordinates of initial point are fixed at $\bar{R}_{\mathrm{d}}=0.25$ and $\bar{C}=-0.075$, and the convergence error is fixed to $10^{-5}$ for the cost function value. It can be seen that the curve decreases rapidly first and then gradually until reaching the convergence towards the minimal value of the bending load. The optimal solution $\bar{F}_{\min }^{S . Q . P}=0.2113$ was reached after eight iterations. If we compare this result with that determined by the first method, it can be noted that this optimal value is $10 \%$ lower than the lowest point of the response surface obtained by the global approach.

Figure 12 displays the convergence of the process parameters towards their optimal values during iterations. We can note a continuous increase in the relative value of die radius until the fourth iteration, and from here onwards, it begins to decrease and ends up being stable. Its optimal value reached is $\bar{R}_{\mathrm{d}}=1.1389$. In the same figure, the evolution of relative clearance is represented during the optimization stage. This parameter converges quickly towards its optimal value since the convergence is attained in four iterations corresponding to its higher limit $\bar{C}=0.15$.

\subsubsection{Minimization of springback}

In the same conditions as mentioned previously, we wish to search the optimal values of process parameters allowing to have a minimum springback. The tolerance of the variation of approximated function for its convergence is chosen equal to $10^{-5}$.The starting point of the algorithm is defined by $\bar{R}_{\mathrm{d}}=1.1875$ and $\bar{C}=0.15$. The evolution of the objective function minimization arround optimal solution is illustrated in Fig. 13. Starting from the initial point, the springback decreases abruptly, then it continues to evolve in more or less continuous way until the fourth iteration, from which it has a constant value. The minimum relative correspond to $\bar{\theta}_{\min }^{S Q P}=2.7778$.

The curve of the variation of the process parameters (relative die radius and relative punch-sheet clearance) versus iteration in minimization step of springback is given in Fig. 14. The value of bending radius evolves rapidly to reach a value nearly constant from the fourth iteration. Its optimal value is $\bar{R}_{\mathrm{d}}=0.25$ which corresponds to its lower limit of its range of variation.

The variation of relative clearance versus cumulated iterations shows a rapid decrease of this parameter until the second iteration then it fluctuates during the next iterations. After convergence, the optimal solution gives a relative value of clearance $\bar{C}=-0.15$. The comparison of the numerical result obtained by this technique with that

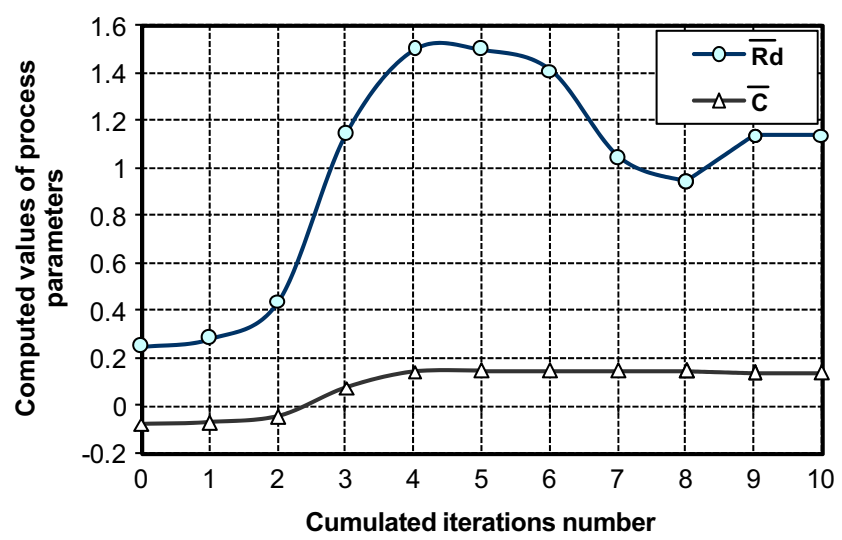

Fig. 12 Evolution of process parameters during the minimization stage of bending load

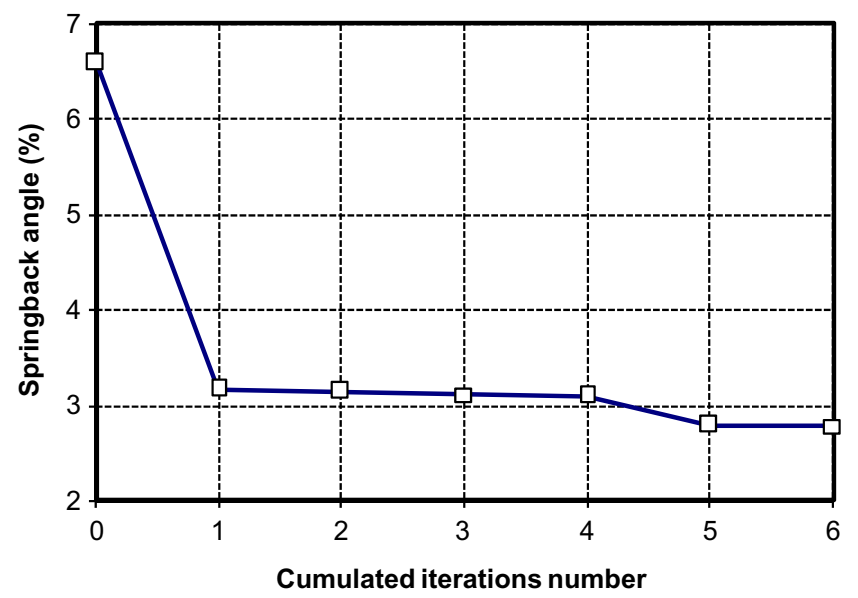

Fig. 13 Evaluation of springback versus iterations number 


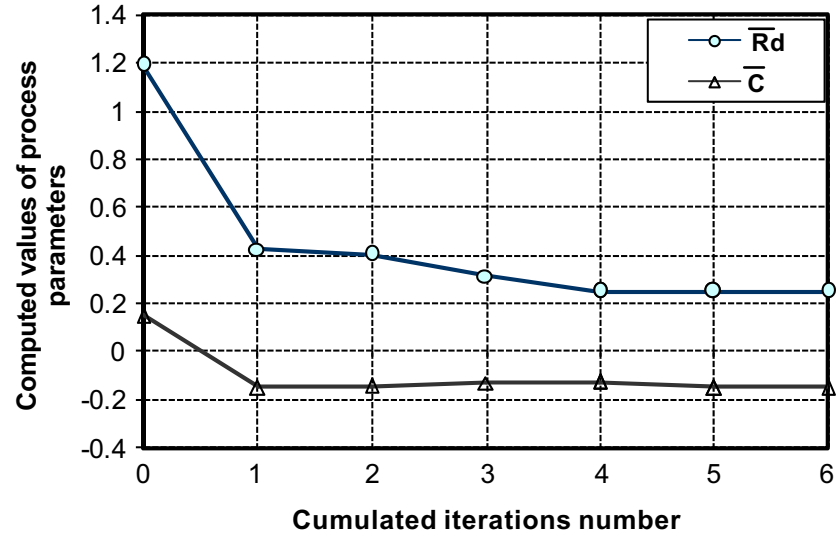

Fig. 14 Variation of process parameters during the optimization of springback

deduced from minimization using global approach presented in Section 5.1 shows a notable difference. Results of this optimization are in conformity as noted by the industrialists, i.e., the bending carried out with small die radii and negative clearances (compression of sheet at the end of the punch stroke) reveals a small springback. It is one of the techniques used to provide parts characterised by good final geometry if we don't take into account the resulting modifications of mechanical behaviour of material in term of damage and stress state.

\subsection{Optimization results obtained using the evolution strategies algorithm}

The third optimization methodology tested in this article is relative to the application of ES technique. One of principal advantages of this evolutionary algorithm is that its process does not require the calculation of objective function gradient and constraints with respect to design variables. Moreover, computation is based on population of individuals, and the convergence can be attained without the use of a mathematical optimization module like the one used in SQP algorithm.

\subsubsection{Minimization of the maximum bending load}

Initially, we want to minimise the bending load. The implementation of the process led to the minimization of the objective function illustrated by Fig. 15. The total number of evaluations is 640 , and the processing time is about $3 \mathrm{~s}$ when using a $2.40-\mathrm{GHz}$ Compaq-based PC. The initial population size was fixed at 400 , and the stopping criteria have been proposed to be equal to $10^{-6}$. We can see in Fig. 15 that the convergence of relative bending load towards its minimal value starts to attain during the first hundred evaluations. In this case, the optimal value obtained is equal to $\bar{F}_{\text {min }}^{\mathrm{ES}}=0.23482$.

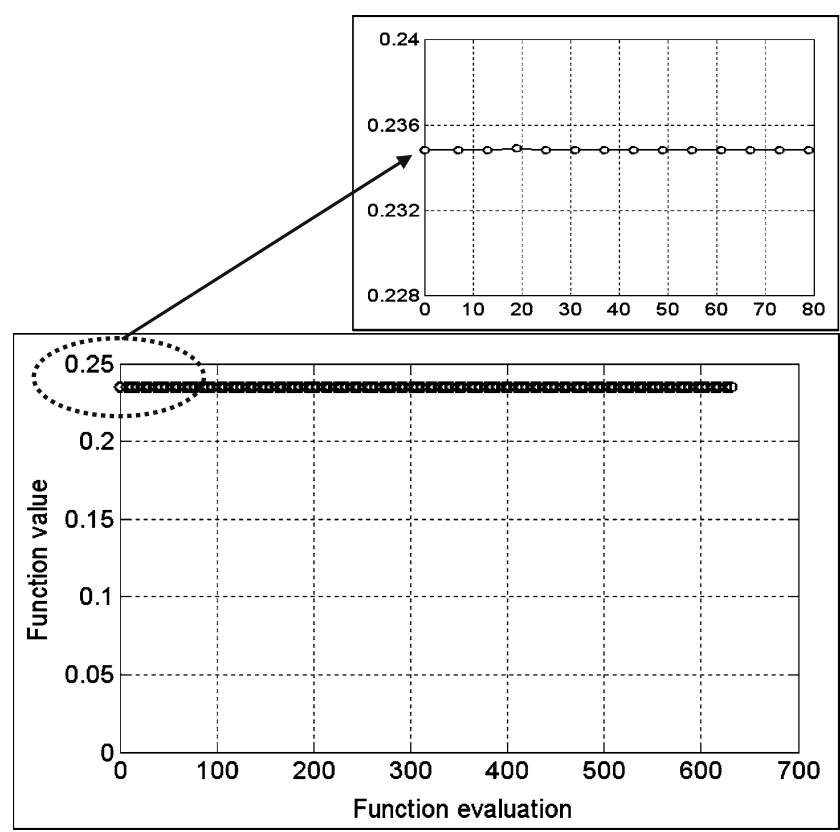

Fig. 15 Convergence history of the relative bending load function with (ES)

Concerning the evolutions of the geometrical process parameters at the same time that the evaluations of the objective function, the algorithm converges to the optimal solution defined by the relative values of die radius and punch-sheet clearance equal to $\bar{R}_{\mathrm{d}}=1.0042$ and $\bar{C}=0.15$, respectively (see Fig. 16). These values can be compared with the previous results to note that they do not differ much from the others.

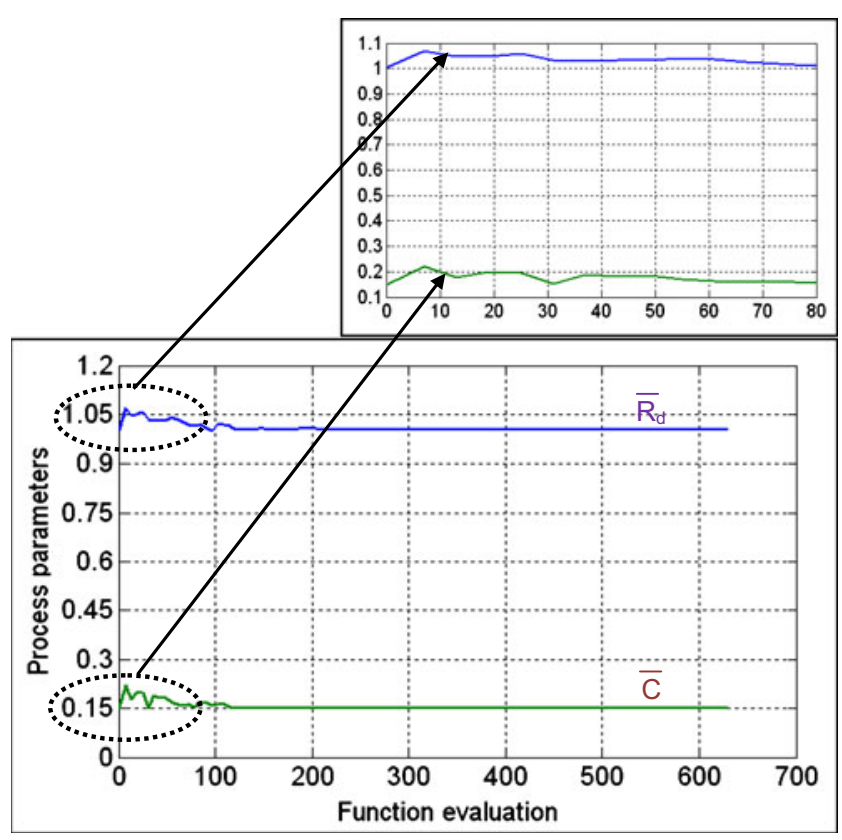

Fig. 16 Convergence of process parameters to the optimal solution with (ES) 


\subsubsection{Minimization of springback}

Secondly, we wish to minimise the cost function of the relative value of springback appearing after removal of the applied loads from the deformed sheet, resulting in the deviation of the product from the applied tooling shape. In this part of work, the application of optimization technique is based on the use of the method of ES.

Figure 17 shows the evolution of the objective function versus a number of evalutions during the optimization process. According to the representative curve, it can be noted that the minimal value of the reponse begins to be stable from the first 120 evaluations.

The global optimum is obtained for a total number of evaluations equal to 760 requiring $4 \mathrm{~s}$ of computing time. The minimal solution corresponds to a relative value of springback of $\bar{\theta}_{\min }^{\mathrm{ES}}=2.9715$. A comparison between this approach and the results obtained by the global approach (see Section 5.1) shows that the two optimal solutions are almost identical, which will make it possible to validate the use of this algorithm.

In Fig. 18, we report the convergence history of relative process parameters versus the number of evalutions during the minimization of the objective function. At the begin of their evolution, each one of these two parameters decreases by presenting weak fluctuations until approximately 150 evaluations, then from here onwards, they will be stabilised. The optimal values of the two variables are $\bar{R}_{\mathrm{d}}=0.25$ and $\bar{C}=-0.0977$, respectively. An excellent agreement is obtained when a comparison of these two results to those

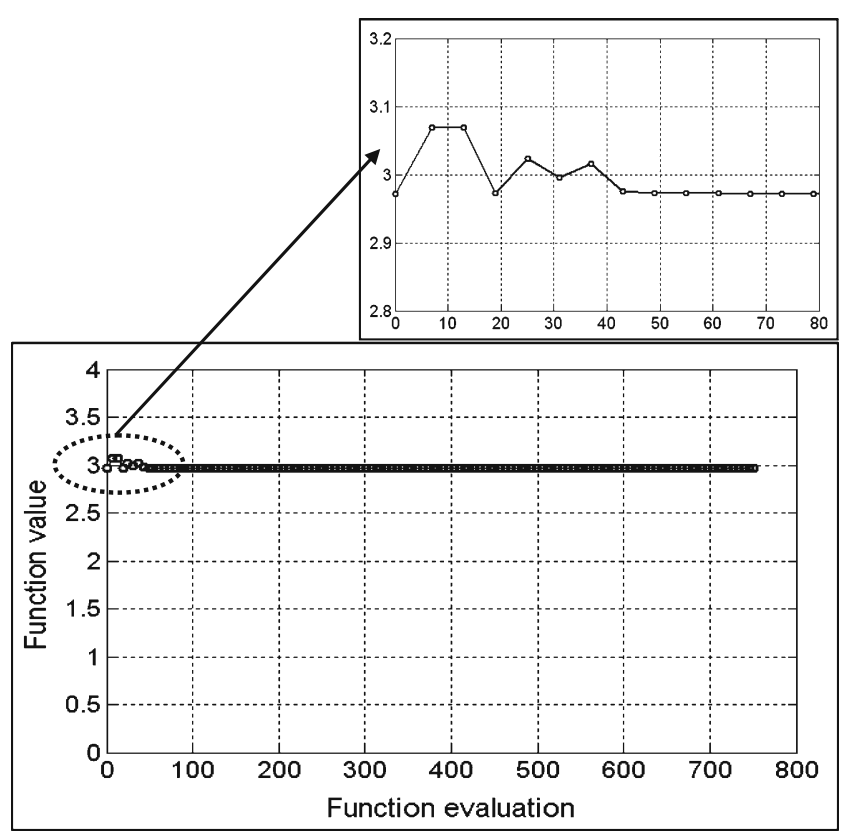

Fig. 17 Convergence history of the relative function of springback with (evolution strategies)

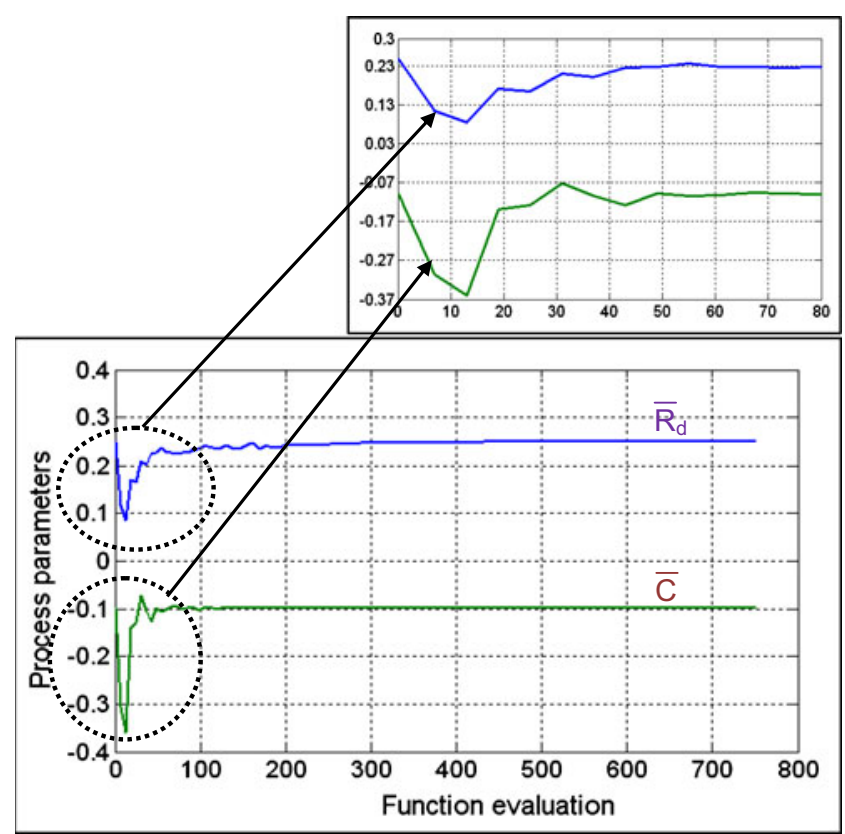

Fig. 18 Variation of relative process parameters during the minimization of the objective function

which are determined by the global approach is carried out. The two methods lead exactly to the same values of the objective function since the optimal values of geometrical parameters are located in the two presented cases at the limits of their intervals of variation.

\subsection{Comparison between optimization results}

In this part of work, one undertakes a comparative study between all results provided by the three optimization methods described previously in order to ensure the validity of each one of them for an optimal prediction of minimal values of bending load and springback. Table 4 recapitulates the obtained results of optimization for the two objective functions studied, $\bar{F}_{\text {min }}$ and $\bar{\theta}_{\text {min }}$, and the deduced values of process parameters. The three optimization techniques: "a global approach", a local approach using RSM based on "SQP algorithm" and "ES" are subscripted $(.)^{\text {Global }},(.)^{\text {S.Q.P }}$, and (.) $)^{\mathrm{ES}}$, respectively.

It can be seen in Table 4 that the numerical results obtained by the ES algorithm of $\bar{F}_{\text {min }}$ and $\bar{\theta}_{\text {min }}$ are very close to the computed values by using global approach which is considered as reference method. Consequently, these results can thus be observed as satisfactory. For SQP algorithm, the maximum relative errors of these two responses compared to global optimization are $10 \%$ and $6 \%$ for $\bar{F}_{\min }$ and $\bar{\theta}_{\min }$, respectively. It can also be noted that the three methods used lead to reduced numerical values of clearance and die radius which are comparable and very close. 
Table 4 Comparison of different results obtained by three optimization methods

\begin{tabular}{lllllll}
\hline Objective functions & $\bar{F}_{\text {min }}^{\text {Global }}$ & $\bar{F}_{\text {min }}^{S Q P}$ & $\bar{F}_{\text {min }}^{\mathrm{ES}}$ & $\bar{\theta}_{\min }^{\text {Global }}$ & $\bar{\theta}_{\text {min }}^{S Q P}$ & $\bar{\theta}_{\text {min }}^{\mathrm{ES}}$ \\
\hline Optimal values & 0.2348 & 0.2113 & 0.23482 & 2.9715 & 2.7778 & 2.9715 \\
$\bar{R}_{d \_ \text {opt }}$ & 1 & 1.1389 & 1.0042 & 0.25 & 0.25 & 0.25 \\
$\bar{C}_{\text {_opt }}$ & 0.15 & 0.1412 & 0.15 & -0.099 & -0.15 & -0.0977 \\
\hline
\end{tabular}

Figure 19 illustrates the variation of minimal values of the bending force after optimization stage versus the methods used. The three values of each of the two parameters are also reported here. We can also see that the optima are predicted at the same design points of tools, and that in the ranges of variation of $R_{\mathrm{d}}$ and $C$, the optimal values are

$\left.\begin{array}{l}R_{\mathrm{d}}^{\text {mean }}=1.0477 \times 4=4.1908 \mathrm{~mm} \\ C^{\text {mean }}=0.14706 \times 4=0.5882 \mathrm{~mm}\end{array}\right\}$ (the avarage values of the three results $)$.

The variations of minimal values of springback after optimization operation according to the method used are reported in Fig. 20. The same figure shows the solutions found corresponding to the three values of these two parameters which make it possible to minimise the spring- back. In this case, the optimal values are predicted at the same points, but they are different from that recommended for the bending load. In fact, the optimal values of die shoulder radius $R_{\mathrm{d}}$ and punch-sheet clearance $C$ located in their intervals of variation are, respectively:

$\left.\begin{array}{l}R_{\mathrm{d}}^{\text {mean }}=0.25 \times 4=1 \mathrm{~mm} \\ C^{\text {mean }}=-0.1155 \times 4=-0.462 \mathrm{~mm}\end{array}\right\}$ (the avarage values of the three results $)$.

In conclusion, the obtained results provide information on the relationship between the two objective functions presented by the maximum bending load and springback. The two optimal solutions are obtained for different values. They are reached for the geometrical process parameters characterised by high values of die radius and clearance in the case of the minimization of bending load and, however,

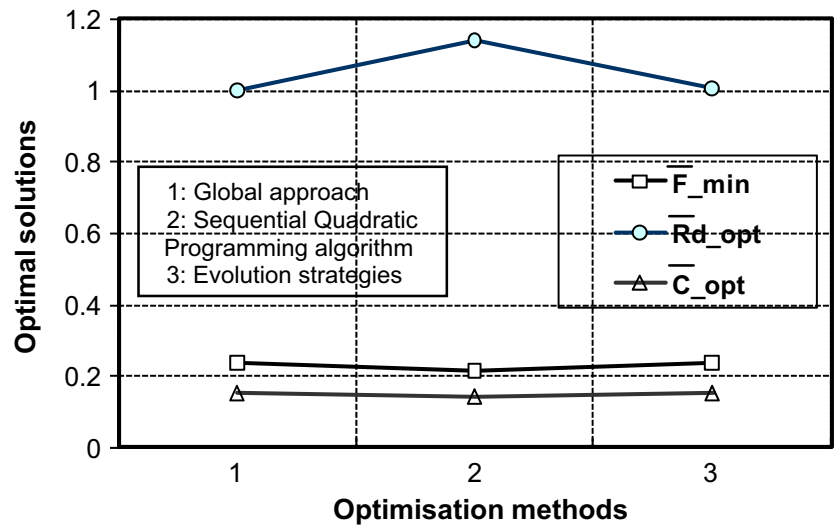

Fig. 19 Representation of the optimal solution of maximum bending load versus used optimization methods by very small values in the second objective function optimization. It can be noted that the most severe conditions given by the smallest die radii $R_{\mathrm{d}}$ and negative clearances $C$ are unrealistic because they involve a cracking of specimens. In this case, significant shearing occurs between punch and sheet, which leads to a decrease in the resistance of the material. In relation with the multi-

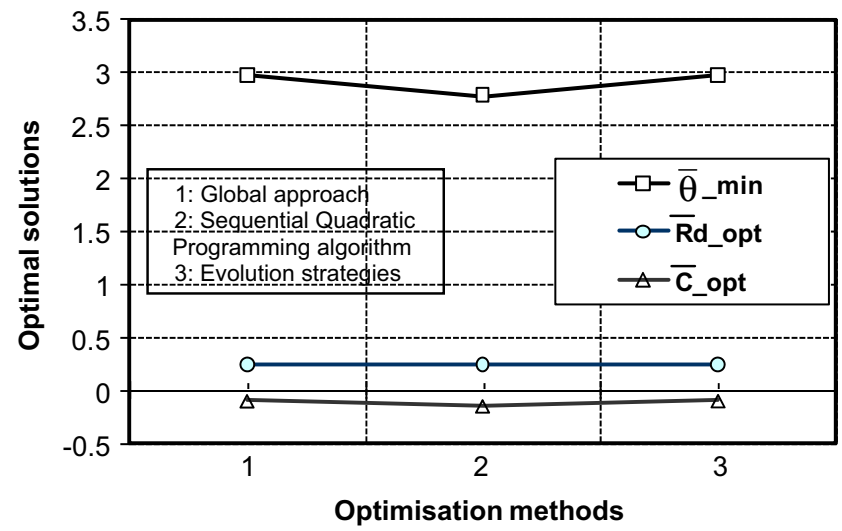

Fig. 20 Representation of the optimal solution of springback versus used optimization methods 
objective optimization, it is thus necessary to restrict the interval of variation of die radii between 2 and $6 \mathrm{~mm}$ and to adopt a small positive values of clearnaces if we wish to obtain resistant parts without defects.

\section{Conclusions}

The mechanical and geometrical quality of parts manufactured by means of a wiping die bending process depends on the choice of the main process parameters, i.e., the die radius and the clearance between the sheet metal and the tools. In order to minimise the responses represented by $F_{\max }$ (maximum punch force), and $\theta$ (springback during unloading), an experimental approach based on the use of (DoE) technique is investigated. A comparison between three optimization methods is done in this work. More specifically, the results obtained by two algorithms: An RSM based on the SQP and an ES algorithm show good agreement with a global approach. However, for the SQP approximation, we observed that the choice of the initial point plays a crucial role in the convergence towards the optimal solution. The search of the optimum was based on the minimization of an objective function, which takes into account of geometrical constraints. In this case, the SQP is more accurate and gives a solution better than those given by the other methods (the values of the objective functions given by SQP are less than those given by GA and ES).

As an example, the optimization algorithms were applied to optimise the die shoulder radius and punch-sheet clearance in order to minimise the maximum bending load and springback. In the case of the minimization of bending load, the optimum determined by all methods is obtained approximately for the same value of the relative clearance $\bar{C}=0.147$ and for slightly different values of the die radius. The maximum value of the relative error does not exceed $10 \%$. It was found that the ES is a powerful optimization tool with respect to its robustness and its convergence speed. Moreover, the major advantage of this algorithm is that it does not require the gradient to be calculated. Concerning the optimization of springback, good agreement was found between the results determined using the global approach and the ES. The minimal values calculated by each of these optimization techniques lead to almost identical values for the process parameters given by $\bar{R}_{\mathrm{d}}=0.25$ and $\bar{C}=-0.09$.

In conclusion, the results determined by the three methods: "a global approach (GA)", "a local approach" using RSM based on SQP, and "ES" allowed to show a good coherence between them and the reliability of each one of these algorithms used.
Acknowledgements The authors gratefully acknowledge the support of Deville SA Company in providing the blank material and material specification. They also thank Dr. Bellett Daniel and Fadte Girish for their helpful comments and suggestions.

Open Access This article is distributed under the terms of the Creative Commons Attribution Noncommercial License which permits any noncommercial use, distribution, and reproduction in any medium, provided the original author(s) and source are credited.

\section{References}

1. Xu L, Reinikainen T, Ren W, Ping Wang B, Han Z, Agnoafer D (2004) A simulation-based multiobjective design optimisation of electronic packages under thermal cycling and bending. Microelectron Reliab 44:1977-1983

2. Wu HC, Altan T (2004) Process optimisation in stamping - a case study for flanging a clutch hub from steel plate. J Mater Process Technol 146:8-19

3. Oral S, Darendeliler H (1997) The optimum die profile for the cylindrical bending of plates. J Mater Process Technol 70:151-155

4. Naceur H, Guo YQ, Batoz JL (2004) Blank optimization in sheet metal forming using an evolutionary algorithm. J Mater Process Technol 151:183-185

5. Bahloul R (2005) Optimisation du procédé de pliage sur presses de pièces en tôles à Haute Limite d'Élasticité. Thèse de doctorat à l'École Nationale Supérieure d'Arts et Métiers d'Angers. In French: Optimisation of the bending process of High Strength Low Alloy sheet metal

6. Tekiner Z (2004) An experimental study on the examination of springback of sheet metals with several thicknesses and properties in bending dies. J Mater Process Technol 145:109-117

7. Inamdar MV, Date PP, Desai UB (2000) Studies on the prediction of springback in air vee bending of metallic sheets using an artificial neural network. J Mater Process Technol 108:45-54

8. Kleijnen JPC (2005) An overview of the design and analysis of simulation experiments for sensitivity analysis. Eur J Oper Res 164:287-300

9. Şehirlioğlu AK, Özler C (2008) The use of mixture experiments in tolerance allocation problems. Int J Adv Manuf Technol 35:769777

10. Ben Ayed L, Delamézière A, Batoz JL, Knopf-Lenoir C (2005) Optimization of the blankholder force distribution in deep drawing. In Proc of the first invited COST 526 Conference on Automatic Process Optimization in Materials Technology, Morschach, Switzerland, pp 229-235

11. Romero VJ, Swiler LP, Giunta AA (2004) Construction of response surface based on lattice-sampling experimental designs with application to uncertainty propagation. Struct Saf 26:201-219

12. Sacks J, Welch WJ, Mitchell TJ, Wynn HP (1989) Design and analysis of computer experiments. Stat Sci 4(4):409-435

13. Simpson TW, Maurey TM, Korte JJ, Mistree F (1998) Comparison of response surface and kriging models for multidisciplinary design optimization. In Proc of the seventh Americain Institute of Aeronautics and Astronautics/USAF/NASA/ISSMO Symposium on Multidisciplinary Analysis \& Optimisation, St. Louis, 4755, pp 1-11

14. Sakata S, Ashida F, Zako M (2003) Structural optimization using Kriging approximation. Comput Methods Appl Mech Eng 192:923-939

15. Bahloul R, Mkaddem A, Dal Santo Ph, Potiron A (2006) Sheet metal bending optimisation using response surface method, 
numerical simulation and design of experiments. Int J Mech Sci 48(9):991-1003

16. Benyounis KY, Olabi AG, Hashmi MSJ (2005) Optimizing the laser-welded butt joints of medium carbon steel using RSM. J Mater Process Technol 164(165):986-989

17. Bahloul R, Dal Santo Ph, Potiron A (2005) Optimisation of process parameters in wiping die bending operation in order to minimise of stresses and Lemaitre's damage. In Proc ESAFORM'05 International Conference on Material Forming, ClujNapoca, Romania, pp 159-162

18. Barthelemy JFM, Haftka RT (1993) Approximation concepts for optimum structural design - a review. Struct Optim 5:129-144

19. Haftka RT, Scott EP (1996) Optimization and experiments-a survey. In: Tatsumi T, Watanabe E, Kambe T (eds) Proceedings of the XIX International Congress of Theoretical and Applied Mechanics. Kyoto, Japan, pp 303-321

20. Box GEP, Wilson KB (1951) On the experimental attainment of optimum conditions. J R Stat Soc B13:1-45

21. Hosder S, Watson LT, Grossman B, Mason WH, Kim H, Haftka RT, Cox SE (1998) Polynomial response surface approximations for the multidisciplinary design optimization of high speed civil transport. Technical Reports of NCSTRL at Virginia Tech CS(TR-01-03)

22. Myers RH, Montgomery DC (2002) Response surface methodology: process and product optimization using designed experiments, 2nd edn. Wiley, New York

23. Roux WJ, Stander N, Haftka RT (1998) Response surface approximations for structural optimization. Int J Numer Methods Eng 42:517-534

24. Kurtaran H, Eskandarian A, Marzougui D, Bedwi NE (2002) Crashworthiness design optimization using successive response surface approximations. Comput Mech 29:409-421

25. Stander N (2001) The successive response surface method applied to sheet-metal forming. In Proc of the first MIT Conference on Computational Fluid and Solid Mechanics, Boston, pp 481-485

26. Kleiber M, Knabel J, Rojek J (2002) Reliability assessment in metal forming operations. In Proc of the Fifth World Congress on Computational Mechanics (WCCMV), Vienna, Austria, pp 39-40

27. Lancster P, Salkauskas K (1986) An introduction: curve and surface fitting. Academic, San Diego

28. Breitkopf P, Rassineux A, Villon P (2002) An introduction to moving least squares meshfree methods. Rev Europ Elem Finis 11:825-868

29. Breitkopf P, Rassineux A, Villon P, Saannouni K, Cherouat H (2001) Meshfree operators for consistent field transfer in large deformation plasticity. In Proc of ECCOMAS-ECCM, Cracow, Poland, pp 275

30. Rassineux A, Breitkopf P, Villon P (2003) Simultaneous surface and tetrahedron mesh adaptation using mesh free techniques. Int $\mathrm{J}$ Numer Meth Engng 57:371-389

31. Krishnamurthy T (2003) Response surface approximation with augmented and compactly supported radial basis functions. In Proc of the forty fourth AIAA/ASME/ASCE/AHS/ASC Struc- tures, Structural Dynamics, and Materials Conference, Norfolk, Virginia, pp 7561

32. McDonald DB, Grantham WJ, Tabor WL, Murphy MJ (2000) Response surface model development for global/local optimization using radial basis functions. In Proc of the eighth American Institute of Aeronautics and Astronautics AIAA 2000-4776/ USAF/NASA/ISSMO Symposium on Multidisciplinary Analysis \& Optimisation, Long Beach, California, pp 1-9

33. Hansen N, Ostermeier A (2001) Completely derandomized selfadaptation in evolution strategies. Evol Comput 9(2):159-195

34. Hansen N, Ostermeier A (1997) Convergence properties of evolution strategies with the derandomized covariance matrix adaptation: The $\left(\mu / \mu_{\mathrm{I}}, \lambda\right)$-CMA-ES. In Proc of the fifth European Congress on Intelligent Techniques and Soft Computing, Aachen, Germany, pp 650-654

35. Hansen N, Müler SD, Koumoutsakos P (2003) Reducing the time complexity of the derandomized evolution strategy with Covariance Matrix Adaptation (CMA-ES). Evol Comput 11(1): $1-18$

36. Bahloul R, Ben Eléchi S, Dal Santo Ph, Naceur H, Potiron A (2006) Optimisation of bending process by means of response surface and moving least squares methods. Far East J Appl Math 22(1):25-54

37. Livatyali H, Altan $T$ (2001) Prediction and elimination of springback in straight flanging using computer aided design methods. Part 1: Experimental investigations. J Mater Process Technol 117:262-268

38. Park DH, Yarlagadda P (2008) Effects of punch load for elliptical deep drawing product of automotive parts. Int J Adv Manuf Technol 35:814-820

39. Bahloul R, Ben Eléchi S, Potiron A (2006) Optimisation of springback predicted by experimental and numerical approach by using reponse surface methodology. J Mater Process Technol 173:101-110

40. Naceur H, Guo YQ, Ben-Elechi S (2006) Response surface methodology for design of sheet forming parameters to control springback effects. Comput Struct 84:1651-1663

41. Breitkopf P, Rassineux A, Savignat JM, Villon P (2004) Integration constraint in diffuse element method. Comput Methods Appl Mech Eng 193:1203-1220

42. Häussler-Combe U, Korn C (1998) An adaptive approach with the Element-Free-Galerkin method. Comput Methods Appl Mech Eng 162:203-222

43. The Math Works Inc (2002) MATLAB Reference Guide. The Math Works Inc, Natick

44. Bahloul R, Dal Santo Ph, Potiron A (2004) Shape optimisation of automotive security-parts regarding the influence of residual stresses and material damage. In Proc of the fourth European Congress on Computational Methods in Applied Sciences and Engineering, Finland, pp 490 